\title{
Analysis and Accurate Numerical Solutions of the Integral Equation Derived from the Linearized BGKW Equation for the Steady Couette Flow
}

\author{
Shidong Jiang ${ }^{\mathrm{a}}$, Li-Shi Luo ${ }^{\mathrm{b}, \mathrm{c}, *}$ \\ ${ }^{a}$ Department of Mathematics Sciences, New Jersey Institute of Technology, \\ Newark, New Jersey 07102, USA \\ ${ }^{b}$ Computational Science Research Center, Beijing 100193, China \\ ${ }^{c}$ Department of Mathematics and Statistics, Old Dominion University, \\ Norfolk, VA 23529, USA
}

\begin{abstract}
The integral equation for the flow velocity $u(x ; k)$ in the steady Couette flow derived from the linearized Bhatnagar-Gross-Krook-Welander kinetic equation is studied in detail both theoretically and numerically in a wide range of the Knudsen number $k$ between 0.003 and 100.0. First, it is shown that the integral equation is a Fredholm equation of the second kind in which the norm of the compact integral operator is less than 1 on $L^{p}$ for any $1 \leq p \leq \infty$ and thus there exists a unique solution to the integral equation via the Neumann series. Second, it is shown that the solution is logarithmically singular at the endpoints. More precisely, if $x=0$ is an endpoint, then the solution can be expanded as a double power series of the form $\sum_{n=0}^{\infty} \sum_{m=0}^{\infty} c_{n, m} x^{n}(x \ln x)^{m}$ about $x=0$ on a small interval $x \in(0, a)$ for some $a>0$. And third, a high-order adaptive numerical algorithm is designed to compute the solution numerically to high precision. The solutions for the flow velocity $u(x ; k)$, the stress $P_{x y}(k)$, and the half-channel mass flow rate $Q(k)$ are obtained in a wide range of the Knudsen number $0.003 \leq k \leq 100.0$; and these solutions are accurate for at least twelve significant digits or better, thus they can be used as benchmark solutions.
\end{abstract}

Keywords: Boltzmann equation, linearized BGKW equation, integral

\footnotetext{
* Corresponding author.

Email addresses: shidong.jiang@njit.edu (Shidong Jiang), lluo@odu.edu (Li-Shi Luo)
}

Preprint submitted to Journal of Computational PhysicsMay 1, Nov. 8, 2015; April 8, 2016 
equation with end-point singularities, Couette flow, Knudsen layer

\section{Introduction}

In small scale devices, such as microfluidics, gaseous flows may become highly nonequilibrium, hence it is required to solve kinetic equations (cf. reviews $[1,2]$, monographs $[3,4,5,6,7,8]$, and relevant references therein) or even molecular dynamics (cf. [9]). Kinetic equations evolve in phase space $(\boldsymbol{x}, \boldsymbol{\xi})$, where $\boldsymbol{\xi}$ is the particle velocity, thus numerical solutions of kinetic equations are far more challenging and demanding than numerical solutions of hydrodynamic equations in physical space $\boldsymbol{x}$. In addition, the collision term in the Boltzmann equation is an integral operator in a five-dimensional space, evaluation of which requires considerable computational effort. To mitigate the difficulty due to the complexity of the collision integral, one often considers the linearized Boltzmann equation or simpler model kinetic equations, such as the Bhatnagar-Gross-Krook-Welander (BGKW) equation $[10,11]$. However, the simplification of the collision term does not alleviate the challenging demand of computing the distribution function in phase space.

There are several canonical flows which have been studied for a long time, including Kramers' flow, Poiseuille flow, and Couette flow; and there has been a systematic and persistent effort to obtain accurate numerical solutions of the kinetic equations corresponding to these flows $[12,13,14,15,16,17,18$, $19,20,21,22,23,24,25,26,27,28]$. Accurate solutions of these canonical flows have been used as benchmarks to test numerical schemes for more general flows $[22,23,24,25,28]$.

For the aforementioned canonical flows, the linearized BGKW equation with the Maxwell diffusive boundary conditions [29, 4] leads to Fredholm integral equations of the second kind with weakly singular kernels, and it has been a continuous effort to obtain accurate numerical solutions of the integral equations for the canonical flows [22, 23, 24, 25, 28, 30, 31, 32]. However, it appears that some advanced modern techniques developed in the last few decades for solving integral equations with singularities at the endpoints have yet to be fully utilized in the community of rarefied gas dynamics (RGD). Indeed, accurately solving these integral equations requires at least two apparatuses: (a) high-order quadratures to evaluate the involved singular integrals and functions and (b) proper discretization schemes to capture the singularities of the solution at the endpoints. Currently, the popular 
approaches for solving these integral equations use the Meijer $G$-function to compute the singular kernels [25, 28], apply smooth Gaussian quadrature to evaluate the singular integrals, and represent the solution with some global expansion of smooth functions or piecewise polynomials on equispaced subintervals. First, the use of Meijer $G$-function for the evaluation of the singular kernel function (e.g., the Abramowitz function in many cases) is very likely to suffer from catastrophic cancellation for large values of the argument, as observed previously [33]. Second, the direct application of a smooth Gaussian quadrature to evaluate the singular integrals either results in a very low accuracy or demands an excessively large number of nodes required for the quadrature. Third and finally, the representation of the solution by using globally smooth polynomial expansion or piecewise polynomials on uniform, equispaced subintervals makes it difficult to capture the singularities of the solution. Thus, the predominant methodologies currently employed in the RGD community fail to obtain high-precision benchmark solutions of these integral equations with a reasonable computational effort [33].

The shortcomings of the aforementioned approaches are especially evident and apparent in the case of obtaining high-precision benchmark solutions of the linearized BGKW equation for canonical flows [22, 23, 24, 25, 28]. For example, Loyalka and Tompson [25] use the Nyström method with GaussKronrod quadrature to solve the integral equation for Kramers' problem. They are only able to obtain the flow velocity with a precision of about 7 digits even though the largest quadrature order is 1312 and a precision of 60 digits is required for the arithmetic computation. More recently, Yap and Sader [28] use the same technique with Gauss-Legendre quadrature to study steady and oscillating Couette flows, and with a large linear system of size $12,800^{2}=163,840,000$ and the arithmetics with 30 digits, they are only able to obtain the velocity between 5 and 8 significant digits for the Knudsen number $k$ between 0.01 and 100.0, respectively. While requiring a significant computational effort in terms of both the multiple-precision arithmetics and large size of quadratures, these popular approaches [25, 28] can only attain a precision far less than the IEEE 64 bit double-precision.

Perhaps the most accurate result for the integral equation for the steady Couette flow to date is obtained by $\mathrm{Li}$ et al. [32] with the Chebyshev collocation method, in which the singular kernel function, i.e., the Abramowitz function, is approximated by its Chebyshev expansion [34], as opposed to the use of the Meijer $G$-function in the previous work [25, 28]. By choosing a proper and accurate approximation for the singular kernel, the work of 
Li et al. [32] greatly improves the accuracy of the solution while significantly reduces the computational effort. However, the work of Li et al. [32] does not employ any advanced modern techniques for numerical solution of the integral equations with singularities at the endpoints, which is the task of the present work.

As mentioned previously, the Couette flow is one of the canonical flows which play an important role for understanding the transport phenomena in gaseous flows in micro-scales (cf., e.g., [6, 9] and references therein). In particular, the Knudsen layer formed at a solid boundary is of great interest. In this paper, we will study the integral equation which is derived from the linearized BGKW equation for the steady Couette flow $[3,4,5,6,7,8]$ :

$$
\begin{aligned}
& u(x)-\frac{1}{k \sqrt{\pi}} \int_{-1 / 2}^{1 / 2} J_{-1}\left(\frac{|x-y|}{k}\right) u(y) \mathrm{d} y=f(x), \\
& f(x):=\frac{1}{2 \sqrt{\pi}}\left[J_{0}\left(\frac{1 / 2-x}{k}\right)-J_{0}\left(\frac{1 / 2+x}{k}\right)\right],
\end{aligned}
$$

where the parameter $k$ is the Knudsen number and $J_{n}$ is the $n$-th order Abramowitz function defined by

$$
J_{n}(x)=\int_{0}^{\infty} t^{n} e^{-t^{2}-x / t} \mathrm{~d} t, \quad n \geq-1
$$

It is easy to show that the kernel function $J_{-1}(|x|)$ has both absolute value and logarithmic singularities at $x=0$, that is,

$$
J_{-1}(|x|)=\phi_{-1}(x)+g_{-1}(x)|x|+h_{-1}(x) \ln |x|,
$$

where $\phi_{-1}, g_{-1}$, and $h_{-1}$ are functions admitting a power series representation about $x=0$. Then, by its definition (1b), the function $f(x)$ on the right-hand side of (1a) is logarithmically singular at both endpoints $x= \pm 1 / 2$.

In what follows, we will first analyze in detail the singularities of the solution of (1a) at the endpoints. We will show that, when the left endpoint $x=-1 / 2$ is shifted to the origin, the solution of (1a) contains singular terms $(x \ln x)^{n}$ for $n \in \mathbb{N}:=\{1,2, \ldots\}$. We would like to point out that while the leading order singularity $x \ln x$ in the solution $u(x)$ of (1a) has been observed previously [1, 35], higher-order singular terms (i.e., $(x \ln x)^{n}$ for $\left.n>1\right)$ do not seem to have been revealed in the literature. We then apply a highorder adaptive collocation method to solve (1a) numerically. Our numerical 
method has the following features. First, in order to capture the singularities of the solution and the right-hand-side function $f(x)$, the collocation points are chosen adaptively with finer and finer mesh spacing toward the endpoints. Second, in order to evaluate the involved integrals accurately, a high-order generalized Gaussian quadrature is applied to compute weakly singular integrals and an automatic adaptive Gaussian quadrature is used to compute nearly singular or smooth integrals to high precision. This way, we are able to provide 12-digit benchmark solutions with a rather modest computational cost, which is considerably less than what is required in the previous work [25, 28, 32].

The remainder of the paper is organized as follows. Section 2 contains some analytic properties of the Abramowitz function and some integral formulas involving logarithmic and monomials, which will be used our theoretical analysis. Section 3 summarizes our main theoretical results, which fully reveal the mathematical properties of the solution $u(x)$. Section 4 describes the numerical method used to solve (1a). Section 5 presents the numerical results for the flow velocity $u(x ; k)$, the stress $P_{x y}(k)$, and the half-channel mass flow rate $Q(k)$ with a wide range of Knudsen number $0.003 \leq k \leq 100.0$, all of which are accurate for at least twelve significant digits. Finally, Section 6 concludes this paper. Appendix A provides the the quadrature weights and abscissas which are used in our calculations, so that our results can be reproduced.

\section{Mathematical Preliminaries}

For the sake of completeness and convenience, we collect in this section some known analytic properties of the Abramowitz function $J_{n}$ and list some formulas for certain definite and indefinite integrals involving logarithmic and monomials, which will be used in the proofs of Theorems 1 and 2, and Corollary 2 in Sec. 3.

The Abramowitz function $J_{n}$ satisfies the following differential and recursive relationships $[36,37]$ :

$$
\begin{aligned}
& J_{n+1}^{\prime}(x)=-J_{n}(x), \\
& 2 J_{n}(x)=(n-1) J_{n-2}(x)+x J_{n-3}(x) .
\end{aligned}
$$

Also, $J_{n}$ can be written in the following form:

$$
J_{n}(x)=f_{n}(x)+x g_{n}(x)+x^{n+1} h_{n}(x) \ln (x), \quad n \geq-1,
$$


where $f_{n}, g_{n}$, and $h_{n}$ are all power series of $x^{2}$ about $x=0$ with $h_{n}(0) \neq 0$. The above formula and its asymptotic expansions in both small and large $x$ are used to evaluate of $J_{n}(x)$ [37]. In particular, we have:

$$
2 J_{1}(x)=\sum_{m=0}^{\infty}\left(a_{m} \ln x+b_{m}\right) x^{m}
$$

with $a_{0}=a_{1}=0, a_{2}=1$, and $b_{0}=-1, b_{1}=\sqrt{\pi}, b_{2}=3(\gamma-1) / 2$,

$$
a_{m}=\frac{2 a_{m-2}}{m(m-1)(m-2)}, b_{m}=\frac{2 b_{m-2}+\left(3 m^{2}-6 m+2\right) a_{m}}{m(m-1)(m-2)}, \quad m \geq 3,
$$

and $\gamma=0.5772156649015328606 \cdots$ is Euler's constant.

The following properties of $J_{n}$ follow from the definition (2) of $J_{n}$ and (4a) and will be used in Section 3.

1. $J_{n}(x)>0$ for any $x>0$;

2. $J_{n}(x)$ is monotonically decreasing on $[0, \infty)$;

3. $J_{n}(x)$ is convex on $[0, \infty)$;

4. $J_{0}(0)=\sqrt{\pi} / 2$.

The following definite and indefinite integrals involving logarithmic and monomials will be used in the proofs of Corollary 1.

$$
\begin{aligned}
& \int x^{n}(\ln x)^{m} \mathrm{~d} x=x^{1+n} m ! \sum_{i=0}^{m} \frac{(-1)^{m-i}(\ln x)^{i}}{(m-i) !(1+n)^{m+1-i}}, \quad m, n \geq 0, \\
& \int x^{-1}(\ln x)^{m} \mathrm{~d} x=\frac{1}{m+1}(\ln x)^{m+1}, \\
& \int x^{-n}(\ln x)^{m} \mathrm{~d} x=x^{1-n} m ! \sum_{i=0}^{m} \frac{(\ln x)^{i}}{(m-i) !(1-n)^{m+1-i}}, \quad m \geq 0, n>1
\end{aligned}
$$




$$
\begin{aligned}
& \int_{0}^{a}|x-y|(x-y)^{2 j} y^{n}(\ln y)^{m} \mathrm{~d} y=\sum_{i=0}^{2 j+1} a_{i} x^{i}+x^{2 j+n+2} \sum_{i=0}^{m} b_{i}(\ln x)^{i}, \\
& \int_{0}^{x} y^{n}(\ln y)^{m} \ln |x-y| \mathrm{d} y=\frac{x^{n+1}(\ln x)^{m+1}}{(n+1)}+x^{n+1} \sum_{i=1}^{m} \alpha_{i}(\ln x)^{i}, \\
& \int_{x}^{a} y^{n}(\ln y)^{m} \ln |x-y| \mathrm{d} y=-\frac{m x^{n+1}(\ln x)^{m+1}}{(n+1)(m+1)}+x^{n+1} \sum_{i=0}^{m} \beta_{i}(\ln x)^{i}+\sum_{j=0}^{\infty} \zeta_{j} x^{j}, \\
& \int_{0}^{a} y^{n}(\ln y)^{m} \ln |x-y| \mathrm{d} y=\frac{x^{n+1}(\ln x)^{m+1}}{(n+1)(m+1)}+x^{n+1} \sum_{i=0}^{m} \gamma_{i}(\ln x)^{i}+\sum_{j=0}^{\infty} \zeta_{j} x^{j},
\end{aligned}
$$

where $0<x<a-\epsilon<a \leq 1, j, m, n \geq 0$; and $a_{i}, b_{i}, c_{i}, \alpha_{i}, \beta_{i}, \gamma_{i}=\left(\alpha_{i}+\beta_{i}\right)$, and $\zeta_{j}$ are all constant coefficients.

Proof of identities (8) - (10). Identity (8a) can be found in the handbook by Gradshteyn and Ryzbik [38, p. 234] and identity (8b) follows by a simple change of variable. Identity (8c) is obtained by applying the following integration by parts formula $m$ times

$$
\int x^{-n}(\ln x)^{m} \mathrm{~d} x=-\frac{x^{1-n}(\ln x)^{m}}{(n-1)}+\frac{m}{(n-1)} \int x^{-n}(\ln x)^{m-1} \mathrm{~d} x .
$$

To prove (9), we expand

$$
(x-y)^{2 j}=\sum_{i=0}^{2 j} C_{2 j}^{i} x^{i}(-1)^{2 j-i} y^{2 j-i}=\sum_{i=0}^{2 j} C_{2 j}^{i} x^{i}(-1)^{i} y^{2 j-i},
$$

pull out $x^{i}$ from the integration in (9), and note that the following integral is finite:

$$
\int_{0}^{a} y^{2 j-i+n}(\ln y)^{m} \mathrm{~d} y, \quad(2 j-i+n)>0, m \geq 0 .
$$


To prove (10a), we expand the integral as follows:

$$
\begin{aligned}
& \int_{0}^{a}|x-y|(x-y)^{2 j} y^{n}(\ln y)^{m} \mathrm{~d} y \\
= & \int_{0}^{x}(x-y)^{2 j+1} y^{n}(\ln y)^{m} \mathrm{~d} y-\int_{x}^{a}(x-y)^{2 j+1} y^{n}(\ln y)^{m} \mathrm{~d} y \\
= & \sum_{i=0}^{2 j+1} C_{2 j+1}^{i} x^{i}(-1)^{2 j+1-i}\left(\int_{0}^{x}-\int_{x}^{a}\right) y^{2 j+1-i+n}(\ln y)^{m} \mathrm{~d} y .
\end{aligned}
$$

Using (8a), we have

$$
\left(\int_{0}^{x}-\int_{x}^{a}\right) y^{2 j+1-i+n}(\ln y)^{m} \mathrm{~d} y=2 x^{2 j+2-i+n} \sum_{l=0}^{m} c_{l}(\ln x)^{m-l}-C,
$$

where $C$ is a constant. Substitution (13) into (12) leads to (10a).

To prove (10b), we write $\ln (x-y)=\ln x+\ln (1-y / x)$ and make the change of variable $z=y / x$, then

$$
\begin{gathered}
\int_{0}^{x} y^{n}(\ln y)^{m} \ln |x-y| \mathrm{d} y=\int_{0}^{1}[\ln x+\ln (1-z)] x^{n+1} z^{n}(\ln x+\ln z)^{m} \mathrm{~d} z \\
=x^{n+1}\left[\sum_{j=0}^{m} C_{m}^{j}(\ln x)^{j+1} \int_{0}^{1} z^{n}(\ln z)^{m-j} \mathrm{~d} z\right. \\
\left.+\sum_{j=0}^{m} C_{m}^{j}(\ln x)^{j} \int_{0}^{1} z^{n}(\ln z)^{m-j} \ln (1-z) \mathrm{d} z\right]
\end{gathered}
$$

thus (10b) follows, since each definite integral on the right side of (14) is finite.

To prove (10c), denote the integral on the left hand side of (10c) by $I_{n, m}$, then the following recurrence relationship can be easily shown by integration by parts:

$$
(1+n) I_{n, m}=(a \ln a)^{n}(a-x) \ln (a-x)+n x I_{n-1, m}+m x I_{n-1, m-1}-m I_{n, m-1} .
$$

Thus, the evaluation of $I_{n, m}$ boils down to that of $I_{0, m}$ and $I_{n, 0}$. The integral $I_{n, 0}$ can be easily obtained by application of (15) $n$ times with $m=0$.

To evaluate $I_{0, m}$, we first use the following identity with $y \geq x$ :

$$
\ln |x-y|=\ln (y-x)=\ln y+\ln \left(1-\frac{x}{y}\right)=\ln y-\sum_{n=1}^{\infty} \frac{1}{n} x^{n} y^{-n},
$$


substitution of the above identity into (10c) with $n=0$ leads to:

$$
\int_{x}^{a}(\ln y)^{m} \ln |x-y| \mathrm{d} y=\int_{x}^{a}(\ln y)^{m+1} \mathrm{~d} y-\sum_{n=1}^{\infty} \frac{x^{n}}{n} \int_{x}^{a} y^{-n}(\ln y)^{m} \mathrm{~d} y .
$$

The integrals on the right hand side of (16) can be computed by using (8a), $(8 \mathrm{~b})$, and (8c), and the special case of (10c) with $n=0$ is proved by noting that the end result consists of the sum of two terms: The first term is a product of $x$ and a $(m+1)$-th order polynomial of $\ln x$, and the second one is a convergent power series of $x$ (about $x=0$ ) for $0<x \leq a-\epsilon<a$ for $\epsilon \in(0, a)$.

Finally, the combination of (10b) and (10c) leads to (10d).

The following corollary is an immediate consequence of (10d) and will be used in the proof of Theorem 2 in Sec. 3.

Corollary 1. Suppose that the functions $K(x)$ and $f(x)$ all admit a power series representation about $x=0$ on $[0, a]$. Then

$$
\begin{aligned}
& \int_{0}^{a} K(x-y) \ln |x-y| f(y) \mathrm{d} y=r(x) x \ln x+s(x), \\
& \int_{0}^{a} K(x-y) f(y) y^{n}(\ln y)^{n} \ln |x-y| \mathrm{d} y=x^{n+1} \sum_{m=0}^{n+1} p_{m}(x)(\ln x)^{n+1-m}+q(x),
\end{aligned}
$$

where $n>0$, and the functions $r(x), s(x), p_{m}(x)$, and $q(x)$ all admit a power series representation (about $x=0$ ) on $[0, a-\epsilon]$ for $\epsilon \in(0, a)$.

Proof. Identity (17a) follows from (10d) by setting $m=0$ and (17b) follows from (10d) directly.

\section{Main Theoretical Results}

We rewrite (1a) in the following standard form

$$
(\mathbf{I}-\mathbf{K}) u=f
$$


where $f$ is defined in $(1 \mathrm{~b}), \mathbf{I}$ is the identify operator, and the operator $\mathbf{K}$ : $L^{p}(I) \rightarrow L^{p}(I)$, for $1 \leq p \leq \infty$, is defined as the following:

$$
[\mathbf{K} u](x)=\int_{I} K(x, y) u(y) \mathrm{d} y, \quad K(x, y):=\frac{1}{k \sqrt{\pi}} J_{-1}\left(\frac{|x-y|}{k}\right),
$$

with $I=[-1 / 2,1 / 2]$. The $L^{p}$ operator norm of $\mathbf{K}$ is defined by

$$
\|\mathbf{K}\|_{p}=\sup _{f \in L^{p}(I)}\left\{\frac{\|\mathbf{K} f\|_{p}}{\|f\|_{p}}: f \neq 0\right\} \text {. }
$$

In particular (cf. Ch. 2 in [39]),

$$
\|\mathbf{K}\|_{1}:=\max _{y \in I} \int_{I}|K(x, y)| \mathrm{d} x, \quad\|\mathbf{K}\|_{\infty}:=\max _{x \in I} \int_{I}|K(x, y)| \mathrm{d} y .
$$

Our main theoretical results concerning the integral equation (1a), or equivalently (18), are summarized in Theorems 1 and 2 and Corollary 2. Theorem 1 proves the existence and uniqueness of the solution. Theorem 2 and Corollary 2 reveal the exact nature of the singularities of the solution at the endpoints.

Theorem 1. For any $k>0$ and $1 \leq p \leq \infty$,

1. $\mathbf{K}$ is a compact operator from $L^{p}(I)$ to $L^{p}(I)$;

2. $\|\mathbf{K}\|_{p} \leq\left[1-J_{0}(1 / 2 k) / J_{0}(0)\right]<1$;

3. The integral operator $(\mathbf{I}-\mathbf{K})$ is invertible and its bounded inverse is given by the Neumann series $(\mathbf{I}-\mathbf{K})^{-1}=\sum_{n=0}^{\infty} \mathbf{K}^{n}$;

4. The integral equation $(\mathbf{I}-\mathbf{K}) u=f$ has a unique solution for any $f \in$ $L^{p}(I)$.

Proof. It can be seen from (3) that $J_{-1}(|x-y| / k)$ is only singular at $x=y$ with the logarithmic singularity at the worst (the absolute value singularity is weaker). So the kernel of $\mathbf{K}$, defined by (19), is a continuous kernel of order 0 and thus $\mathbf{K}$ is compact on $L^{p}(I)$ to $L^{p}(I)$ for any $1 \leq p \leq \infty$ (cf. [40, p. 123]). 
We now compute $\|\mathbf{K}\|_{\infty}$ explicitly as follows:

$$
\begin{aligned}
\|\mathbf{K}\|_{\infty} & =\max _{x \in I} \int_{I}|K(x, y)| \mathrm{d} y \\
& =\frac{1}{k \sqrt{\pi}} \max _{x \in I} \int_{I} J_{-1}\left(\frac{|x-y|}{k}\right) \mathrm{d} y \\
& =\frac{1}{k \sqrt{\pi}} \max _{x \in I}\left[\int_{-1 / 2}^{x} J_{-1}\left(\frac{x-y}{k}\right) \mathrm{d} y+\int_{x}^{1 / 2} J_{-1}\left(\frac{y-x}{k}\right) \mathrm{d} y\right] \\
& =\frac{1}{\sqrt{\pi}} \max _{x \in I}\left[\left.J_{0}\left(\frac{x-y}{k}\right)\right|_{-1 / 2} ^{x}-\left.J_{0}\left(\frac{y-x}{k}\right)\right|_{x} ^{1 / 2}\right] \\
& =\frac{2}{\sqrt{\pi}} \max _{x \in I}\left\{J_{0}(0)-\frac{1}{2}\left[J_{0}\left(\frac{1+2 x}{2 k}\right)+J_{0}\left(\frac{1-2 x}{2 k}\right)\right]\right\} \\
& =\frac{2}{\sqrt{\pi}}\left[J_{0}(0)-J_{0}(1 / 2 k)\right]=1-\frac{J_{0}(1 / 2 k)}{J_{0}(0)} \\
& <1,
\end{aligned}
$$

where the first inequality follows from the convexity of $J_{0}$ and the last inequality follows from the monotonicity of $J_{0}$ and the property (5) of $J_{n}$ in Sec. 2.

Since the kernel of the operator $\mathbf{K}$ is symmetric, $\|\mathbf{K}\|_{1}=\|\mathbf{K}\|_{\infty}$. And the result for general $p \in(1, \infty)$ follows from generalized Young's inequality (cf. $[40$, p. 9$])$. It then follows naturally that $(\mathbf{I}-\mathbf{K})$ is invertible and its inverse is represented by a convergent Neumann series (cf. [41, Ch. 2]). Hence, the integral equation (18) has a unique solution for any $f \in L^{p}(I)$ for $p \in[1, \infty]$.

The next theorem concerns the singularities of the solution of (1a) at the endpoints $x= \pm 1 / 2$. The singularities of the solution at both endpoints are identical due to the symmetry of the flow, thus we only need to study the singularity at one endpoint, say, at $x=-1 / 2$. We first make a simple change of variable to move the interval $[-1 / 2,1 / 2]$ to $[0,1 / k]$ so (1a) becomes

$$
u(x)-\frac{1}{\sqrt{\pi}} \int_{0}^{1 / k} J_{-1}(|x-y|) u(y) \mathrm{d} y=\frac{1}{2 \sqrt{\pi}}\left[J_{0}\left(\frac{1}{k}-x\right)-J_{0}(x)\right] .
$$

We will still use $\mathbf{K}$ to denote the integral operator on the left-hand side and $f$ to denote the inhomogeneous term on the right-hand side of (23). 
Theorem 2. Define $F_{n}(x):=\left[\mathbf{K}^{n} f\right](x)$, and

$$
a_{0}=\frac{1}{2} \min \left(\frac{1}{k}, 1\right), a_{n+1}=\left[1-\frac{1}{4(n+1)^{2}}\right] a_{n}, \forall n \in \mathbb{N}_{0}:=\{0,1,2, \ldots\} .
$$

Then for $k>0$ and $0<x<a_{n}$,

$$
F_{n}(x)=q_{n}(x)+\sum_{m=1}^{n+1} p_{m}(x)(x \ln x)^{m}
$$

where $q_{n}(x)$ and $p_{m}(x)$ admit a power series about $x=0$. That is, for each $n, F_{n}(x)$ has new singular terms $p_{n+1}(x)(x \ln x)^{n+1}$, of which the new leading singular term is $(x \ln x)^{n+1}$. Hence, the solution $u(x)$ of $(23)$ can be represented by the following convergent double power series (about $x=0$ ):

$$
u(x)=\sum_{n=0}^{\infty} \sum_{m=0}^{\infty} c_{n, m} x^{n}(x \ln x)^{m}, \quad 0 \leq x<a_{\infty}:=\frac{2}{\pi} a_{0} .
$$

Proof. From Theorem 1, the solution $u(x)$ can be written in terms of a convergent Neumann series:

$$
u(x)=\sum_{n=0}^{\infty}\left[\mathbf{K}^{n} f\right](x):=\sum_{n=0}^{\infty} F_{n}(x)
$$

thus (25) is a simple consequence of (24) and the fact that

$$
a_{\infty}(k)=a_{0}(k) \prod_{n=1}^{\infty}\left(1-\frac{1}{4 n^{2}}\right)=\frac{2}{\pi} a_{0}(k) .
$$

We now show by induction that (24) is true.

The first term in the Neumann series is simply the right hand side function f, i.e.,

$$
F_{0}(x)=f(x)=\frac{1}{2 \sqrt{\pi}}\left[J_{0}\left(\frac{1}{k}-x\right)-J_{0}(x)\right]
$$


and by (5) it is of the form $f_{0}(x)+x g_{0}(x)+(x \ln x) h_{0}(x)$ with $h_{0}, f_{0}, g_{0}$ all power series of $x^{2}$ (about $\left.x=0\right)$ on $\left(0, a_{0}\right)$. So $(24)$ is true for $n=0$. Assume now that

$$
F_{n-1}(x)=q_{n-1}(x)+\sum_{m=0}^{n} p_{m}(x)(x \ln x)^{m}, \quad \text { for } 0<x<a_{n-1} .
$$

We will show that $F_{n}(x)$ is of the form (24) for $0<x<a_{n}$.

By definition,

$$
F_{n}(x):=\left[\mathbf{K} F_{n-1}\right](x):=\int_{0}^{1 / k} K(x, y) F_{n-1}(y) \mathrm{d} y .
$$

We now split the above integral into two parts:

$$
\begin{aligned}
F_{n}(x) & =\underbrace{\int_{0}^{a_{n-1}} K(x, y) F_{n-1}(y) \mathrm{d} y}_{\Psi_{1}}+\underbrace{\int_{a_{n-1}}^{1 / k} K(x, y) F_{n-1}(y) \mathrm{d} y}_{\Psi_{2}} \\
: & =\Psi_{1}(x)+\Psi_{2}(x) .
\end{aligned}
$$

First, the kernel $K(x, y)$ is real analytic for $x \in\left[0, a_{n}\right]$ and $y \in\left[a_{n-1}, 1 / k\right]$ since the two intervals are disjoint due to the fact $a_{n}<a_{n-1}$. Thus $\Psi_{2}(x)$ admits a power series (about $x=0$ ) on $\left[0, a_{n}\right]$.

Consider $\Psi_{1}(x)$ now. Since $K(x, y)=J_{-1}(|x-y|) / \sqrt{\pi}$, by $(5)$ we have

$$
K(x, y)=K_{1}(x-y)+K_{2}(x-y)|x-y|+K_{3}(x-y) \ln |x-y|,
$$

where $K_{i}, i=1,2,3$, are functions which admit a power series representation about $x=0$. We can split $\Psi_{1}(x)$ further into three parts

$$
\begin{aligned}
\Psi_{1}(x):= & L_{1}(x)+L_{2}(x)+L_{3}(x) \\
= & \underbrace{\int_{0}^{a_{n-1}} K_{1}(x-y) F_{n-1}(y) \mathrm{d} y}_{L_{1}(x)} \\
& +\underbrace{\int_{0}^{a_{n-1}} K_{2}(x-y)|x-y| F_{n-1}(y) \mathrm{d} y}_{L_{2}(x)} \\
& +\underbrace{\int_{0}^{a_{n-1}} K_{3}(x-y) \ln |x-y| F_{n-1}(y) \mathrm{d} y}_{L_{3}(x)} .
\end{aligned}
$$


It follows from the induction assumption on $F_{n-1}$ and (9) that $L_{1}(x)$ has a power series representation (about $x=0$ ) on $\left[0, a_{n}\right]$. And by (10a), the most singular term in $L_{2}(x)$ is $x^{n+2}(\ln x)^{n}$. Also, by (17a), (17b) and the induction assumption, $L_{3}(x)$ contains all singular terms in $F_{n-1}$ and the new term $(x \ln x)^{n+1} p_{n+1}(x)$.

Finally, (24) is proved by combining the above results.

Remark 1. $F_{n}(x)$ has logarithmic singularity at both endpoints. Thus $(24)$ does not hold on the entire interval $[0,1 / k]$. This is the reason why we need to use a smaller interval $\left[0, a_{n}\right]$ for each $F_{n}$.

Theorem 2 does not guarantee that the coefficients $c_{0, m}$ with $m \geq 1$ in (25) be nonzero. The following Corollary shows that all leading singular terms $(x \ln x)^{m}$ are present so long as the first leading singular term $(x \ln x)$ appears in the solution.

Corollary 2. The coefficients $c_{m}:=c_{0, m}$ of the leading singular terms $(x \ln x)^{m}$ in the solution $u(x)$ given by the double power series of (25) are fully determined by $u(0)$ as follows:

$$
c_{m}=-\frac{1}{k \sqrt{\pi} m^{2}} c_{m-1}, \quad c_{1}=-\frac{1}{k \sqrt{\pi}}\left[\frac{1}{2}+u(0)\right], \quad m>1 .
$$

Proof. From the properties of $J_{n}$ given in Section 2, we have

$$
J_{0}(x)=f_{0}(x)+(x \ln x) h_{0}(x), \quad h_{0}(0)=1 .
$$

Then by its definition (1b), the function $f$ in the right hand side of (18) has the $x \ln x$ term with the coefficient $-1 /(2 k \sqrt{\pi})$ and has no higher order singular terms $(x \ln x)^{m}$ for $m>1$. From the properties of $J_{-1}$, it can seen that the most singular term in the kernel $K(x, y)$ is $-(\ln |x-y|) /(k \sqrt{\pi})$.

By (10d), it can be shown that the only contribution for $(x \ln x)^{m}$ by $[\mathbf{K} u](x)$ is due to the term

$$
\int_{0}^{1} \ln |x-y| c_{m-1}(y \ln y)^{m-1} \mathrm{~d} y=\frac{1}{m^{2}} c_{m-1}(x \ln x)^{m}, \quad m \geq 1 .
$$


By matching the singular terms $(x \ln x)^{m}$ on both sides of $(18)$, for $m=1$, and since $c_{0}=u(0)$, we obtain

$$
c_{1}+\frac{1}{k \sqrt{\pi}} u(0)=-\frac{1}{2 k \sqrt{\pi}} .
$$

For $m>1$, we have

$$
c_{m}+\frac{1}{k \sqrt{\pi}} \frac{1}{m^{2}} c_{m-1}=0 .
$$

Rearranging (31) and (32), we obtain (28).

With the theorems and corollary stated above, the mathematical nature of the integral equation (1a) and its solution are completely understood.

Remark 2. Several earlier publications on the Couette flow $[42,13,43]$ mention a work by Willis [44] on the existence and uniqueness of the solution of the linearized BGKW equation. Since it exists only in the form of thesis [44], a brief summary and discussion of this work by Willis is provided below for the sake of readers.

For the steady Couette flow, the perturbation distribution function $\phi\left(\xi_{x}, x\right)$ for the linearized BGKW equation satisfies the following equation:

$$
\xi_{x} \partial_{x} \phi=-\frac{1}{k}(\phi-u), \quad u=\frac{1}{\sqrt{\pi}} \int_{-\infty}^{\infty} \phi e^{-\xi_{x}^{2}} \mathrm{~d} \xi_{x},
$$

where $\xi_{x}$ is the particle velocity along $x$ axis, with the boundary conditions:

$$
\phi\left(\xi_{x} \gtrless 0, x=\mp 1 / 2\right)=\mp 1 .
$$

Integrating (33) with the boundary conditions (34) leads to:

$$
\phi^{ \pm}(x)=\mp \exp \left(-\frac{(x \pm 1 / 2)}{k \xi_{x}}\right)+\frac{1}{k \xi_{x}} \int_{\mp 1 / 2}^{x} \exp \left(-\frac{\left(x \pm x^{\prime}\right)}{k \xi_{x}}\right) u\left(x^{\prime}\right) \mathrm{d} x^{\prime},
$$

where $\phi^{ \pm}(x):=\phi\left(\xi_{x} \gtrless 0, x\right)$. Willis proposed the following iterative scheme to solve (35): setting $u=u_{n-1}\left(x^{\prime}\right)$ in the right-hand side of (35) to obtain $\phi_{n}^{ \pm}(x)$, and showed it to be convergent because:

$$
\begin{aligned}
& \max \left|\phi_{n+1}^{ \pm}-\phi_{n}^{ \pm}\right| \leq \delta_{1} \delta_{2} \max \left|\phi_{n}^{ \pm}-\phi_{n-1}^{ \pm}\right| \\
& \delta_{1}:=1-e^{-1 /\left(k\left|\xi_{x}\right|\right)} \leq 1, \\
& \delta_{2}:=\frac{2}{\sqrt{\pi}} \int_{0}^{\infty}\left(1-e^{-1 /\left(k \xi_{x}\right)}\right) e^{-\xi_{x}^{2}} \mathrm{~d} \xi_{x}<1, \forall k \neq 0 .
\end{aligned}
$$


Because the sequence $\left\{\phi_{n}\right\}$ converges, thus one can prove the existence and uniqueness of the solution $\phi$ of (35), or equivalently (33), obtained by the iterative scheme [44].

Clearly, there is a connection between the convergence of Willis' iterative scheme for the solution of (35) and the convergence of the Neumann series for the solution of (1a) given by Theorem 1. However, the present work reveals the nature of the singularities which has never been seen previously. With his iterative scheme [44], Willis only showed the first-order singularity $|x \mp 1 / 2| \ln |x \mp 1 / 2|$ in $u(x ; k)$ at the walls $x= \pm 1 / 2$.

\section{Numerical Algorithm}

In what follows, we will describe the numerical algorithm to solve (1a), which is characterized by two key features. First, the kernel $J_{-1}(|x-y| / k)$ has both logarithmic and absolute value singularities when $x=y$, as stated in (3). And second, both the right-hand side of (1a), $f(x)$, and the solution $u(x)$ are singular at the endpoints $x= \pm 1 / 2$. To be more precise, if we shift the left endpoint $x=-1 / 2$ to the origin, then the right hand side $f(x)$ is of the form $\phi(x)+g(x) x \ln x$ with the functions $\phi$ and $g$ which admit a power series representation about $x=0$, and the singularities of the solution $u(x)$ are described in detail by (25).

To solve (1a), we will employ some advanced modern techniques for solving integral equations with singular kernel and corner singularities. Since our problem is one-dimensional, it is relatively small in size and the computational speed is not a major concern. To obtain accurate solutions of benchmark quality for the problem, we employ a collocation method in which the collocation points are chosen adaptively with finer and finer mesh spacing toward the endpoints in order to capture the singularity of the solution at the endpoints. The underlying integrals are then computed via either high order generalized Gaussian quadratures for singular functions (cf. e.g., [45, 46, 47]) or an adaptive Gaussian quadrature routine with high precision. We would like to remark here that there have been some recent work on efficient and accurate treatment on corner singularities based on the Nyström method (cf. e.g., $[48,49]$ and references therein), albeit with a slightly lower accuracy.

In the ensuing discussion, the interval $[-1 / 2,1 / 2]$ is mapped to $[0,1]:=$ $I$. The interval $I$ is divided into $N_{m}+2$ equispaced subintervals, and then each end subinterval is refined dyadically into $N_{e}$ smaller and smaller subintervals - the closer to an endpoint, the smaller the subinterval. Altogether, the 
total number of intervals is $N_{s}=N_{m}+2 N_{e}$. For the $p$-th order method, the solution is approximated by a polynomial of degree $(p-1)$ on each subinterval $I_{i}$, and the collocation points on $I_{i}$ are shifted and scaled Legendre nodes from $[-1,1]$ to $I_{i}$. Thus, the total number of collocation points $N$ is $p \times N_{s}$.

The discretization of (1a) leads to the following linear system to be solved:

$$
\mathbf{A} \cdot \mathbf{u}=\mathbf{f}
$$

where $\mathbf{u}$ and $\mathbf{f}$ are the column vectors of length $N$ consisting of the values of the solution $u(x ; k)$ and the right-hand-side function $f(x ; k)$ at the abscissas, respectively, and $\mathbf{A}$ is the interaction matrix of size $N \times N$ to be constructed as follows. The interaction matrix $\mathbf{A}$ can be decomposed into $N_{s} \times N_{s}$ number of $p \times p$ blocks. Denote the $i j$-th block of $\mathbf{A}$ by $[\mathbf{A}]_{i j}$. For off-diagonal blocks of $\mathbf{A}$, i.e., $[\mathbf{A}]_{i j}$ with $i \neq j$, we first use an adaptive Gaussian quadrature routine with a prescribed precision to compute

$$
\begin{aligned}
& v_{m n}:=\int_{I_{j}} K\left(x_{i, m}, y\right) P_{n}(\tilde{y}) \mathrm{d} y, \quad n=0,1, \ldots, p-1, \\
& \tilde{y}:=\left(\frac{2}{b_{j}-a_{j}}\left[y-\frac{a_{j}+b_{j}}{2}\right]\right),
\end{aligned}
$$

where $x_{i, m}$ is the $m$-th collocation point on the subinterval $I_{i}, I_{j}:=\left[a_{j}, b_{j}\right]$, and $P_{n}$ is the Legendre polynomial of degree $n$. Then the $m$-th row of $[\mathbf{A}]_{i j}$ is:

$$
\mathbf{r}_{m}=\mathbf{v}_{m} \cdot \mathbf{T}, \quad \mathbf{v}_{m}:=\left(v_{m 1}, v_{m 2}, \ldots, v_{m p}\right),
$$

where $\mathbf{T}$ is the $p \times p$ Gauss-Legendre transform matrix which converts the function values at the collocation points to the Legendre expansion coefficients. Note that $\mathbf{T}$ only needs to be computed once a priori and stored if necessary. Also, the integrals here are relatively easy to compute numerically because $\left\{x_{i, m}\right\} \notin I_{j}$, that is, the collocation points $\left\{x_{i, m}\right\} \in I_{i}$ are outside the integration interval $I_{j}$.

The diagonal blocks of $\mathbf{A}$ can be computed similarly, though the involved integrals are harder to compute numerically since the integrand is weakly singular, i.e., the integrand has a logarithmic singularity within the interval of integration. In order to reduce the computational cost, we apply generalized Gaussian quadratures to compute diagonal blocks. The procedure is as follows. For each Legendre abscissa $x_{m}, m=1,2, \cdots, p$, on the standard interval $[0,1]$, we apply generalized Gaussian quadrature algorithm to find 
a set of quadrature abscissas $\left\{y_{n}\right\}$ and the corresponding weights $\left\{w_{n}\right\}$ such that the function $f$ can be approximated by the quadrature with a prescribed precision:

$$
\begin{aligned}
& \int_{0}^{1} f(y) \mathrm{d} y \approx \sum_{n=1}^{n_{k}} w_{n} f\left(y_{n}\right), \\
& f(y)=\psi_{1}(y)+\psi_{2}(y)\left|x_{m}-y\right|+\psi_{3}(y) \ln \left|x_{m}-y\right|,
\end{aligned}
$$

where $\psi_{i}, i=1,2,3$, are polynomials of degree less than, say, $3 p$. Then the following integral can also be approximated by the quadrature:

$$
\int_{I_{j}} K\left(x_{j, m}, y\right) u(y ; k) \mathrm{d} y \approx \sum_{n=1}^{n_{m}} \tilde{w}_{n} K\left(x_{j, m}, \tilde{y}_{n}\right) u\left(\tilde{y}_{n} ; k\right),
$$

where $\tilde{w}_{n}$ and $\tilde{y}_{n}$ are scaled weights and (shifted) abscissas, respectively, and $u\left(\tilde{y}_{n}\right)$ can be obtained via Legendre interpolation from $\left\{x_{j, m}\right\}$ to $\left\{\tilde{y}_{n}\right\}$. The weights and abscissas of the generalized Gaussian quadrature to evaluate the singular integrals in (37) are listed in the appendix section so that our results can be easily reproduced.

\section{Numerical Results}

This section presents the evidence to illustrate the performance of the algorithm and provides some benchmark solutions for a wide range of Knudsen number $0.003 \leq k \leq 100.0$. As in Section 4 , the interval is shifted to $[0,1]$ from $[-1 / 2,1 / 2]$. We will also use the notation $u(x ; k)$ instead of $u(x)$ (and similarly for $f(x ; k))$ throughout this section so that the dependence on $k$ is explicitly seen.

We implement the algorithm outlined in the preceding section in Fortran. We used the gfortran compiler (version 4.8.1) with quadruple precision arithmetic on a 64 bit LinUX workstation with a $2.93 \mathrm{GHz}$ INTEL XEON ${ }^{\circledR} \mathrm{CPU}$ which has six cores and $12 \mathrm{MB}$ of cache. We have also used OpenMP to parallelize the computation for constructing the matrix as well as matrix-vector products in GMRES. The linear system is then solved using GMRES which restarts when the number of iteration reaches 100 and terminates when the relative residual falls below $10^{-24}$.

In general, the condition number of the linear system increases as $k$ decreases. With $k=0.003$, the linear system is rather ill-conditioned and it 
takes about 430 iterations for GMRES to converge. We use LiNUX system command time to measure the elapsed time which includes the time for constructing the matrix and for solving the linear system using GMRES. The elapsed time for the entire calculation is about 62.8 seconds and this is the longest time among the cases studied in this work. With $k=10.0$, the linear system is well conditioned and it only takes 10 iterations for GMRES to converge. The elapsed time is only about 23.9 seconds.

\subsection{Endpoint singularities}

To obtain accurate solution for $u(x ; k)$, we must first accurately compute the right-hand side of $(1 \mathrm{a}), f(x ; k)$, which is singular at the endpoints. The properties of $f(x ; k)$ in fact determine that of the solution $u(x ; k)$. The Knudsen layers at channel walls are generated by the singularities of $f(x ; k)$. Figure 1 shows both $f(x ; k)$ and $u(x ; k)$ for $k=0.03,0.1,1.0$, and 10.0. Because both $f(x ; k)$ and $u(x ; k)$ are anti-symmetric about the channel center $x=1 / 2$, Fig. 1 only shows $f(x ; k)$ and $u(x ; k)$ in one half of the flow domain $1 / 2 \leq x \leq 1$.
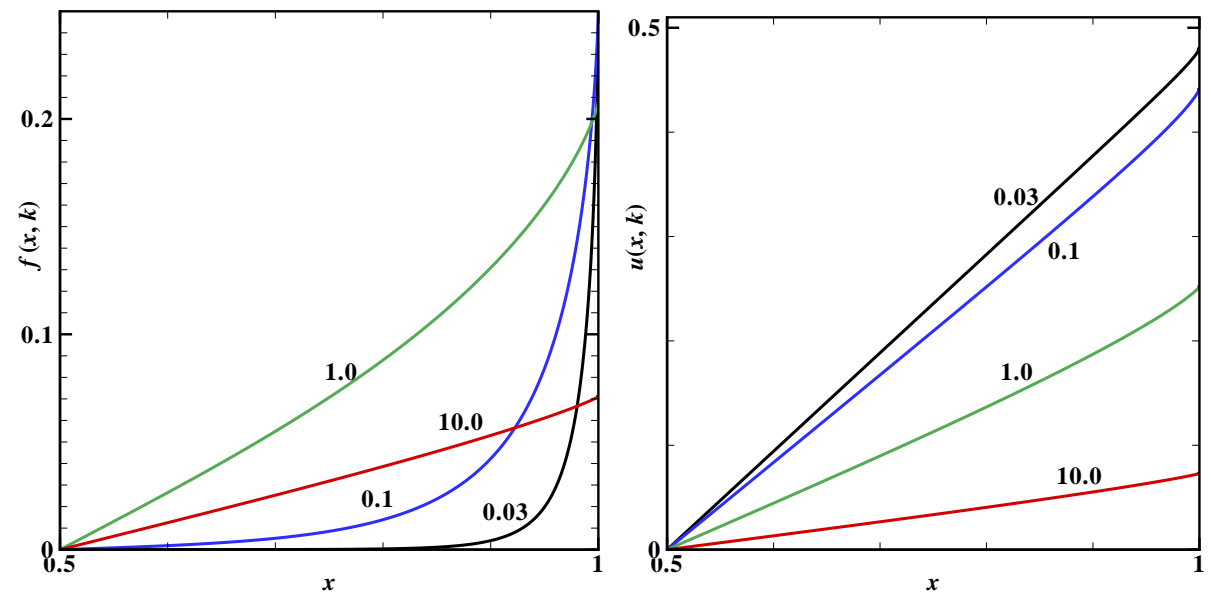

Figure 1: $f(x ; k)$ (left) and $u(x ; k)$ (right) for $k=0.03,0.1,1.0$, and 10.0, with the value of $k$ annotated near the corresponding curves.

It can be shown that near the left endpoint $x=0$, the leading-order asymptotic behavior of the inhomogeneous term in the right-hand side of the integral equation (1a), $f$, is

$$
f(x ; k) \sim \frac{1}{2 \sqrt{\pi}}\left[\frac{(1-3 \gamma)}{2} \frac{1}{k}-\frac{1}{k} \ln \frac{1}{k}+\frac{x}{k} \ln \frac{x}{k}\right],
$$


where it is assumed that $1 \gg k \gg x$. The leading-order singular term of $f(x ; k)$ is $(x / k) \ln (x / k)$ for a fixed $k$.

As shown in Fig. 1, qualitatively, the velocity $u(x ; k)$ appears to be very close to a straight line, which is the solution of the Navier-Stokes equation, and especially so in the vicinity of channel center and for very small and large $k$. Although it is not clearly shown in the figure, it can be proved that the velocity derivative $\mathrm{d} u(x ; k) / \mathrm{d} x \rightarrow \infty$ as $x$ approaches to the endpoints, i.e., the velocity profile $u(x ; k)$, with $k$ fixed, is tangent to the channel walls at the endpoints, similar to the behavior of $f(x)$ at the end point $x=1$ shown in the left panel of Fig. 1 (also, cf. Fig. 3 in [32] and related discussion therein).

The accuracy of the solution of (1a) critically depends on the resolution of the endpoint singularities. To demonstrate that our algorithm can effectively capture the leading-order singularities $(x \ln x)^{m}, m \geq 1$, near the shifted endpoint $x=0$, we compute the solution $u(x ; k)$ in a very small interval $(0, a), a \approx 1.5 \cdot 10^{-7}$, and then fit the solution of $u(x ; k)-u(0 ; k)$ with the following formula:

$$
l(x)=c_{1,0} x+c_{1} x \ln x+c_{2,1} x^{2} \ln x+c_{2}(x \ln x)^{2} .
$$

The numerical solution of $u(x ; k)-u(0 ; k), k=0.03$, is obtained with the number of collocation points $N=2000$. Using 240 points of the numerical solution of $u(x ; k)-u(0 ; k)$ which are closest to the endpoint $x=0$, the $L^{2}$ or the mean squared error of the least-squares fitting with (40) is $3.4 \cdot 10^{-30}$. The numerical results of $u(x ; k)-u(0 ; k)$ with $k=0.03$ and its least-squares fit $l(x)$ are shown in Fig. 2.

The values of the coefficients of $x \ln x$ and $(x \ln x)^{2}$, i.e., $c_{1}$ and $c_{2}$ in (40), computed from (28) are -0.3760160283459086 and 1.767869386997996 , respectively, while the values obtained by the least-squares fitting are -0.37601602637 and 1.749711, respectively. It can be seen that the leading order singular term $x \ln x$ is accurately captured numerically - the numerical value of $c_{1}$ agrees with its analytic value for eight significant digits. This accuracy is maintained for all values of the Knudsen number $k$ used in this work. 




Figure 2: The numerical solution of $u(x ; k)-u(0 ; k)$ with $k=0.03$ and its least-squares fit on a small interval near the origin on log-log scales. The (red) circles and (blue) solid line represent the numerical solution and the least-squares fit $l(x)=-0.6068046 x-$ $0.37601602637 x \ln x+3.409260 x^{2} \ln x+1.749711(x \ln x)^{2}$, respectively.

\subsection{Convergence}

To demonstrate the effectiveness of our solution method, we compute the $L^{2}$ error for the velocity $u(x ; k)$

$$
E\left(u_{N}\right):=\sqrt{\frac{\int_{I}\left\|u_{N}(x ; k)-u_{*}(x ; k)\right\|^{2} \mathrm{~d} x}{\int_{I}\left\|u_{*}(x ; k)\right\|^{2} \mathrm{~d} x}},
$$

where the reference solution $u_{*}(x ; k)$ is obtained with $N=2000$ for $k=0.03$ and $N=1600$ for other values of $k$, i.e., $k=0.1,1.0$, and 10.0, and the integrals are computed by using the Gauss-Legendre quadrature described in Sec. 4. We use $p=10$ in our calculations unless otherwise stated, i.e., the solution is approximated by a polynomial of degree $(p-1)$ on each subinterval $I_{i}$ (cf. Sec. 4 for the details).

Figure 3 shows the $L^{2}$ error $E$ for the velocity $u(x ; k)$ with a few values of the Knudsen number $k$. In all cases shown in Fig. 3, the rate of convergence is about 10, thus the theoretical order of accuracy has been achieved in all calculations.

\subsection{Benchmark solutions}

We now provide the numerical results. In addition to the velocity $u(x ; k)$, we also provide the velocity derivative at the channel center, $u^{\prime}(x=1 / 2 ; k)$. 


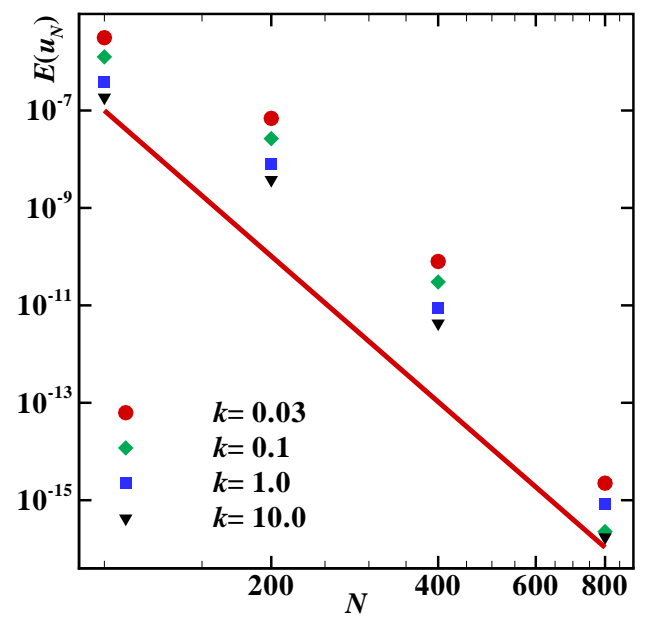

Figure 3: The $N$-dependence of relative $L^{2}$ error $E\left(u_{N}\right)$ defined by (41) for the velocity $u_{N}(x ; k)$ on log-log scale. $N$ is the total number of discretization point. $k=0.03,0.1$, 1.0, and 10.0. The solid line has a slope of -10 , and the designated order of the method is $p=10$.

It can be shown that $u(x=1 ; k) \leq 1 / 2$ and $u^{\prime}(x=1 / 2 ; k) \geq 1$ for $k \geq 0$. The values of the flow velocity at the wall, $u(x=1 ; k)$, and and the velocity derivative at the channel center, $u^{\prime}(x=1 / 2 ; k)$, allow us to define the microscopic slip velocity and the macroscopic slip velocity [6,32], respectively. Table 1 provides the data of $u(x ; k)$ in the upper half channel at $y=0.6,0.7$, $0.8,0.9$, and 1.0, and Table 2 provides the data for velocity at the boundary, $u(x=1 ; k)$ and $u^{\prime}(x=1 / 2 ; k)$; the values of $u(x=1, k)$ are taken from Table 1 with $N=1600$. Apparently, the solution of $u(x ; k)$ converges as the total number of collocation points $N$ increases. It can be seen that the results of $u(x ; k)$ are precise to at least twelve significant digits independent of the values of $x$ and $k$. We note that the present results agree with the ones obtained previously with a different approach [32]. However the present approach requires far less computational effort than the previous one [32] due to the computational efficiency of the high-order adaptive collocation method.

We also compute the stress $P_{x y}(k)$ and the half-channel mass flow rate 
Table 1: The values of the velocity $u_{N}(x ; k)$ at $x=0.6,0.7,0.8,0.9$, and 1.0 , for $0.003 \leq$ $k \leq 100.0$ and $400 \leq N \leq 1600$.

\begin{tabular}{|c|c|c|c|c|c|}
\hline$x$ & 0.6 & 0.7 & 0.8 & 0.9 & 1.0 \\
\hline $\bar{N}$ & & & $k=0.003$ & & \\
\hline 400 & 0.09939398007244217 & 0.1987879601448843 & 0.2981819402173285 & 0.3975759203785835 & 0.4978915175888030 \\
\hline 800 & 0.09939398014204283 & 0.1987879602840856 & 0.2981819404261305 & 0.3975759206569863 & 0.4978915352788807 \\
\hline \multirow[t]{2}{*}{1600} & 0.09939398014207544 & 0.1987879602841508 & 0.2981819404262283 & 0.3975759206571167 & 0.4978915352789693 \\
\hline & & & $k=0.01$ & & \\
\hline 400 & 0.09800810020241383 & 0.1960162013012055 & 0.2940243425786597 & 0.3920355719207134 & 0.4930697754531371 \\
\hline 800 & 0.09800810022280691 & 0.1960162013419917 & 0.2940243426398391 & 0.3920355720023112 & 0.4930697807741956 \\
\hline \multirow[t]{2}{*}{1600} & 0.09800810022280926 & 0.1960162013419964 & 0.2940243426398461 & 0.3920355720023207 & 0.4930697807742208 \\
\hline & & & $k=0.03$ & & \\
\hline 400 & 0.09425510233079511 & 0.1885155964989118 & 0.2828084717603230 & 0.3773525608775003 & 0.4800058665530938 \\
\hline 800 & 0.09425510233708972 & 0.1885155965115045 & 0.2828084717792374 & 0.3773525609029753 & 0.4800058682766751 \\
\hline \multirow[t]{2}{*}{1600} & 0.09425510233708991 & 0.1885155965115049 & 0.2828084717792380 & 0.3773525609029764 & 0.4800058682766829 \\
\hline & & & $k=0.1$ & & \\
\hline 400 & 0.08356104029274617 & 0.1673490502283097 & 0.2518108070975897 & 0.3383684060663754 & 0.4412246405122556 \\
\hline 800 & 0.08356104029425423 & 0.1673490502313504 & 0.2518108071022446 & 0.3383684060729892 & 0.4412246409722400 \\
\hline \multirow[t]{2}{*}{1600} & 0.08356104029425424 & 0.1673490502313504 & 0.2518108071022446 & 0.3383684060729897 & 0.4412246409722421 \\
\hline & & & $k=0.3$ & & \\
\hline 400 & 0.06645430069459216 & 0.1335709509472140 & 0.2023607233387561 & 0.2751706693573815 & 0.3672125694339120 \\
\hline 800 & 0.06645430069493926 & 0.1335709509479253 & 0.2023607233398804 & 0.2751706693590903 & 0.3672125695500499 \\
\hline \multirow[t]{2}{*}{1600} & 0.06645430069493927 & 0.1335709509479253 & 0.2023607233398804 & 0.2751706693590906 & 0.3672125695500504 \\
\hline & & & $k=1.0$ & & \\
\hline 400 & 0.04453194115206441 & 0.08976290005945922 & 0.1366691806914674 & 0.1872336429972648 & 0.2518613399698612 \\
\hline 800 & 0.04453194115212170 & 0.08976290005957814 & 0.1366691806916597 & 0.1872336429975758 & 0.2518613399894732 \\
\hline \multirow[t]{2}{*}{1600} & 0.04453194115212171 & 0.08976290005957814 & 0.1366691806916597 & 0.1872336429975760 & 0.2518613399894732 \\
\hline & & & $k=2.0$ & & \\
\hline 400 & 0.03283175101362668 & 0.06620080866800679 & 0.1008399340224033 & 0.1381797101408611 & 0.1852462993677936 \\
\hline 800 & 0.03283175101364504 & 0.06620080866804505 & 0.1008399340224655 & 0.1381797101409661 & 0.1852462993740218 \\
\hline \multirow[t]{2}{*}{1600} & 0.03283175101364504 & 0.06620080866804505 & 0.1008399340224655 & 0.1381797101409662 & 0.1852462993740218 \\
\hline & & & $k=3.0$ & & \\
\hline 400 & 0.02678842250716726 & 0.05400761302339581 & 0.08223931900196659 & 0.1126006454389917 & 0.1504282444961314 \\
\hline 800 & 0.02678842250717640 & 0.05400761302341487 & 0.08223931900199767 & 0.1126006454390459 & 0.1504282444992069 \\
\hline \multirow[t]{2}{*}{1600} & 0.02678842250717640 & 0.05400761302341487 & 0.08223931900199767 & 0.1126006454390460 & 0.1504282444992075 \\
\hline & & & $k=5.0$ & & \\
\hline 400 & 0.02021810350612312 & 0.04074510549514082 & 0.06199427039072078 & 0.08474655782849507 & 0.1126351880282314 \\
\hline 800 & 0.02021810350612680 & 0.04074510549514850 & 0.06199427039073332 & 0.08474655782851835 & 0.1126351880294590 \\
\hline \multirow[t]{2}{*}{1600} & 0.02021810350612680 & 0.04074510549514850 & 0.06199427039073332 & 0.08474655782851842 & 0.1126351880294592 \\
\hline & & & $k=7.0$ & & \\
\hline 400 & 0.01655896140054647 & 0.03335952222887089 & 0.05072335823656649 & 0.06925336240175322 & 0.09171689613455394 \\
\hline 800 & 0.01655896140054846 & 0.03335952222887504 & 0.05072335823657328 & 0.06925336240176656 & 0.09171689613521612 \\
\hline \multirow[t]{2}{*}{1600} & 0.01655896140054846 & 0.03335952222887504 & 0.05072335823657328 & 0.06925336240176661 & 0.09171689613521435 \\
\hline & & & $k=10.0$ & & \\
\hline 400 & 0.01324840054213267 & 0.02667954575379200 & 0.04053574032131034 & 0.05526789346648103 & 0.07292211299294639 \\
\hline 800 & 0.01324840054213370 & 0.02667954575379414 & 0.04053574032131383 & 0.05526789346648848 & 0.07292211299328582 \\
\hline \multirow[t]{2}{*}{1600} & 0.01324840054213370 & 0.02667954575379414 & 0.04053574032131383 & 0.05526789346648851 & 0.07292211299328496 \\
\hline & & & $k=30.0$ & & \\
\hline 400 & 0.006255046873582013 & 0.01258094337096306 & 0.01907084826563646 & 0.02589565792276850 & 0.03381357342227730 \\
\hline 800 & 0.006255046873582138 & 0.01258094337096332 & 0.01907084826563689 & 0.02589565792276988 & 0.03381357342232957 \\
\hline \multirow[t]{2}{*}{1600} & 0.006255046873582138 & 0.01258094337096332 & 0.01907084826563689 & 0.02589565792276989 & 0.03381357342231838 \\
\hline & & & $k=100.0$ & & \\
\hline 400 & 0.002523722536703575 & 0.005070345851147414 & 0.007669850267518905 & 0.01037682493364896 & 0.01343072948081491 \\
\hline 800 & 0.002523722536703587 & 0.005070345851147439 & 0.007669850267518945 & 0.01037682493364925 & 0.01343072948081877 \\
\hline 1600 & 0.002523722536703587 & 0.005070345851147439 & 0.007669850267518945 & 0.01037682493364925 & 0.01343072948081877 \\
\hline
\end{tabular}

$Q(k)$ :

$$
\begin{aligned}
P_{x y}(k) & =-\frac{1}{\sqrt{\pi}}\left[\frac{2}{k} \int_{1 / 2}^{1} J_{0}((x-1 / 2) / k) u(x ; k) \mathrm{d} x+J_{1}(1 / 2 k)\right], \\
Q(k) & =\int_{1 / 2}^{1} u(x ; k) \mathrm{d} x .
\end{aligned}
$$

It can be shown that $0 \geq P_{x y}(k)>-1 / 2 \sqrt{\pi}$ and $1 / 4 \geq Q(k)>0$ for 
Table 2: The dependence of the velocity at boundary $x=1, u(1 ; k)$, and the velocity derivative at the channel center $x=1 / 2, u^{\prime}(1 / 2 ; k)$, on the Knudsen number $k$. The results are obtained with an adaptive mesh with $N=1600$ (with 96 subintervals of equal length in the middle, 32 dyadic refinements towards each endpoint, and 10 Legendre nodes on each subinterval).

\begin{tabular}{ccc}
\hline$k$ & $u(1 ; k)$ & $u^{\prime}(1 / 2 ; k)$ \\
\hline 0.003 & 0.4978915352789693 & 0.9939398014207545 \\
\hline 0.01 & 0.4930697807742208 & 0.9800810020043015 \\
\hline 0.03 & 0.4800058682766829 & 0.9425456003244014 \\
\hline 0.1 & 0.4412246409722421 & 0.8352857656469417 \\
\hline 0.3 & 0.3672125695500504 & 0.6635300770297120 \\
\hline 1.0 & 0.2518613399894732 & 0.4442284697467991 \\
\hline 2.0 & 0.1852462993740218 & 0.3274745769375018 \\
\hline 3.0 & 0.1504282444992075 & 0.2672070059395808 \\
\hline 5.0 & 0.1126351880294592 & 0.2016944318181617 \\
\hline 7.0 & 0.09171689613521435 & 0.1652086347955840 \\
\hline 10.0 & 0.07292211299328496 & 0.1321955790519311 \\
\hline 30.0 & 0.03381357342231838 & 0.06243844733659697 \\
\hline 100.0 & 0.01343072948081877 & 0.02520098284529616 \\
\hline
\end{tabular}

$k \in[0, \infty)$. The results of both $P_{x y}(k)$ and $Q(k)$ are given in Table 3. Since $Q(k)$ is integrated from $u(x ; k)$, its precision should be comparable to that of $u(x ; k)$ or better. However, the evaluation of $P_{x y}(k)$ involves both the singularities of $J_{0}(x)$ at $x=0$ and $u(x ; k)$ at $x=1 / 2$, thus the evaluation of $P_{x y}$ has a more stringent requirement for quadrature than the that of $Q$.

\section{Conclusions}

In this paper we have studied in detail the integral equation derived from the linearized BGKW equation with the Maxwell diffusive boundary condition for the steady Couette flow. We have proved the existence and uniqueness for the solution of the integral equation. We have shown that the solution of the velocity $u$ near an endpoint can be represented by a convergent double power series (about $x=0$ ) in terms of $x^{m}(\ln x)^{n}$, where $x$ is the distance to the endpoint, and determined the coefficients of the leading order singular terms $(x \ln x)^{n}$ through a recurrence relation, for $n \geq 1$.

We have also developed a high-order numerical algorithm to obtain accurate solution of the integral equation in a wide range of the Knudsen number 
Table 3: The dependence of the stress $P_{x y}$ and the half-channel mass flow rate $Q$ on the Knudsen number $k$.

\begin{tabular}{ccc}
\hline$k$ & $P_{x y}$ & $Q$ \\
\hline 0.003 & $-1.490909702131201 \cdot 10^{-3}$ & $1.242445655299172 \cdot 10^{-1}$ \\
\hline 0.01 & $-4.900405009657547 \cdot 10^{-3}$ & $1.225330275292623 \cdot 10^{-1}$ \\
\hline 0.03 & $-1.413798601526842 \cdot 10^{-2}$ & $1.180147037188893 \cdot 10^{-1}$ \\
\hline 0.1 & $-4.155607782558620 \cdot 10^{-2}$ & $1.057028408172292 \cdot 10^{-1}$ \\
\hline 0.3 & $-9.344983511356682 \cdot 10^{-2}$ & $8.560111699820618 \cdot 10^{-2}$ \\
\hline 1.0 & $-1.694625753368226 \cdot 10^{-1}$ & $5.804708735555459 \cdot 10^{-2}$ \\
\hline 2.0 & $-2.083322536749375 \cdot 10^{-1}$ & $4.281659776113917 \cdot 10^{-2}$ \\
\hline 3.0 & $-2.266437497658084 \cdot 10^{-1}$ & $3.489298506190833 \cdot 10^{-2}$ \\
\hline 5.0 & $-2.446632678455994 \cdot 10^{-1}$ & $2.627042060967383 \cdot 10^{-2}$ \\
\hline 7.0 & $-2.536943539674479 \cdot 10^{-1}$ & $2.147460412330841 \cdot 10^{-2}$ \\
\hline 10.0 & $-2.611624603488405 \cdot 10^{-1}$ & $1.714449048590649 \cdot 10^{-2}$ \\
\hline 30.0 & $-2.743853873277227 \cdot 10^{-1}$ & $8.043009085700258 \cdot 10^{-3}$ \\
\hline 100.0 & $-2.796682147138912 \cdot 10^{-1}$ & $3.226757181742400 \cdot 10^{-3}$ \\
\hline
\end{tabular}

$0.003 \leq k \leq 100.0$. The results include the flow velocity $u(x ; k)$, the stress $P_{x y}(k)$, and the half-channel mass flow rate $Q(k)$, all of which are accurate for at least twelve significant digits. To the best of our knowledge, our results presented in this work are more accurate than any existing data in literature and can be used as benchmark data. Our algorithm is highly efficient. The order of accuracy is 10; and in the worst case of $k=0.003$, the elapsed time for the entire calculation is only about a minute on a computer with six cores.

Our understanding of the endpoint singularity of $u(x ; k)$ can serve as a guide for modeling and simulation of nonequilibrium low-speed flows in which the Knudsen layer plays the crucial role [32]. Clearly, in the context of linearized Boltzmann equation, the Knudsen layer is a phenomenon due to the endpoint singularities in $u(x ; k)$. In other words, one cannot accurately capture the Knudsen layer without resolving the endpoint singularities [32]. Thus one cannot hope to capture the Knudsen layer or its manifestation $a$ priori by using the lattice Boltzmann equation $[50,51,52,53]$ or similar schemes, which is a special case of the linearized BGKW equation in the diffusive limit [54] and is incapable to capture any Knudsen-number effects beyond the Navier-Stokes equations [55, 56, 57].

The analysis and the numerical algorithm used here can be easily extended to analyze and solve the integral equations for Kramers' problem and 
Poiseuille flow. These problems are currently under investigation, and the results will be reported in the future.

\section{Acknowledgments}

The authors would like to thank the anonymous referees for their useful comments and suggestions, which helped improve the presentation of the paper. S. Jiang was supported by the National Science Foundation under grant DMS-1418918 and would like to thank Dr. Zydurnas Gimbutas at NIST for helpful discussions. L.-S. Luo would like to acknowledge the support from the Richard F. Barry Jr. Endowment from Old Dominion University. Part of this work was done in the Summer of 2014 when S. Jiang was visiting the Computational Science Research Center in Beijing, China.

\section{Appendix A. Quadrature nodes and weights for constructing the diagonal blocks of the interaction matrix}

In this appendix, we list the quadrature weights and abscissas for accurately evaluating the integrals of the following form

$$
\begin{aligned}
& \int_{0}^{1} \varphi\left(y ; x_{n}\right) \mathrm{d} y \approx \sum_{i-1}^{N_{q}} w_{i} \varphi\left(y_{i} ; x_{n}\right) \\
& \varphi\left(y ; x_{n}\right)=p_{1}(y)+\left|x_{n}-y\right| p_{2}(y)+p_{3}(y) \ln \left|x_{n}-y\right|
\end{aligned}
$$

where $p_{m}, m=1,2,3$, are polynomials of degree less than 30 , and $x_{n}(n=1$, $2, \ldots, 10)$ is the $n$-th Legendre node on the interval $[0,1]$. These quadrature nodes and weights are used to construct the diagonal blocks of the interaction matrix when discretizing (1a). We list only the nodes and weight for $n=1$, $\ldots, 5$, since the nodes and weights for $n=6, \ldots, 10$, can be obtained by symmetry. 
Table A.4: The quadrature nodes and weights accurate to 20 digits for evaluating the integral of $(\mathrm{A} .1)$ with $\varphi\left(y ; x_{1}\right)$, and $x_{1}=0.130467357414141399610 \cdot 10^{-1}$. The total number of nodes is $N_{q}=33$.

\begin{tabular}{|l|c|}
\hline Nodes & Weights \\
\hline $0.273612974852367768380 \cdot 10^{-3}$ & $0.697159188639274825879 \cdot 10^{-3}$ \\
$0.139812489794156739720 \cdot 10^{-2}$ & $0.152494285680170747031 \cdot 10^{-2}$ \\
$0.324946325453380474357 \cdot 10^{-2}$ & $0.213197817651618622426 \cdot 10^{-2}$ \\
$0.555718717910089599381 \cdot 10^{-2}$ & $0.242590302591390666642 \cdot 10^{-2}$ \\
$0.798133363390943820347 \cdot 10^{-2}$ & $0.236206919268878341353 \cdot 10^{-2}$ \\
$0.101668312577330264011 \cdot 10^{-1}$ & $0.195728487723173970675 \cdot 10^{-2}$ \\
$0.118122051875448364147 \cdot 10^{-1}$ & $0.130438919929371083910 \cdot 10^{-2}$ \\
$0.127516288043818283773 \cdot 10^{-1}$ & $0.582396403529115215563 \cdot 10^{-3}$ \\
$0.130955402368943676360 \cdot 10^{-1}$ & $0.318989294809545966819 \cdot 10^{-3}$ \\
$0.139217029097696913366 \cdot 10^{-1}$ & $0.152585721268974787881 \cdot 10^{-2}$ \\
$0.165149123120158349027 \cdot 10^{-1}$ & $0.385247981430954006623 \cdot 10^{-2}$ \\
$0.220183474544979191195 \cdot 10^{-1}$ & $0.735074493378686513744 \cdot 10^{-2}$ \\
$0.316001572236647671382 \cdot 10^{-1}$ & $0.119971342955652875424 \cdot 10^{-1}$ \\
$0.463572403010820412977 \cdot 10^{-1}$ & $0.176747914242475264005 \cdot 10^{-1}$ \\
$0.672292402992997070455 \cdot 10^{-1}$ & $0.241889503153383312000 \cdot 10^{-1}$ \\
$0.949295270626930393464 \cdot 10^{-1}$ & $0.312848756617644439962 \cdot 10^{-1}$ \\
0.129894321470388716366 & $0.386659797986991125703 \cdot 10^{-1}$ \\
0.172249623347159828416 & $0.460112303782515652436 \cdot 10^{-1}$ \\
0.221795057680864805202 & $0.529915535581842852496 \cdot 10^{-1}$ \\
0.278003571569014726519 & $0.592851145901272809772 \cdot 10^{-1}$ \\
0.340035797408005113311 & $0.645913414865240254862 \cdot 10^{-1}$ \\
0.406767727101099308531 & $0.686434819738653899217 \cdot 10^{-1}$ \\
0.476830096349505052891 & $0.712194165617760576512 \cdot 10^{-1}$ \\
0.548657581388456805000 & $0.721504183525398473162 \cdot 10^{-1}$ \\
0.620545602868418584018 & $0.713275609713352797047 \cdot 10^{-1}$ \\
0.690712256663701917557 & $0.687055307125227898321 \cdot 10^{-1}$ \\
0.757362693088856989254 & $0.643036974724017642607 \cdot 10^{-1}$ \\
0.818753187764629589376 & $0.582044403416407947285 \cdot 10^{-1}$ \\
0.873252230082682834778 & $0.505489029177118042285 \cdot 10^{-1}$ \\
0.919396231292359716010 & $0.415305586528908457209 \cdot 10^{-1}$ \\
0.955937947688746007186 & $0.313872041241292875190 \cdot 10^{-1}$ \\
0.981886567423264858602 & $0.203926571075514119887 \cdot 10^{-1}$ \\
0.996542607381555519820 & $0.886096512672274504975 \cdot 10^{-2}$ \\
\hline
\end{tabular}

\section{References}

[1] E. P. Gross, E. A. Jackson, and S. Ziering. Boundary value problems in kinetic theory of gases. Ann. Phys., 1:141-167, 1957.

[2] M. M. R. Williams. A review of the rarefied gas dynamics theory associated with some classical problems in flow and heat transfer. Z. Angew. Math. Phys., 52(3):500-516, 2001.

[3] M. N. Kogan. Rarefied Gas Dynamics. Plenum Press, New York, 1969. 
[4] C. Cercignani. The Boltzmann Equation and Its Applications. Springer, New York, 1988.

[5] C. Cercignani, R. Illner, and M. Pulvirenti. The Mathematical Theory of Dilute Gases. Springer, New York, 1994.

[6] C. Cercignani. Rarefied Gas Dynamics: From Basic Concepts to Actual Calculations. Cambridge University Press, Cambridge, UK, 2000.

[7] I. N. Ivchenko, S. K. Loyalka, and Jr. R. V. Tompson. Analytical Methods for Problems of Molecular Transport, volume 83 of Fluid Mechanics and its Applications. Springer, Dordrecht, The Netherlands, 2007.

[8] Y. Sone. Molecular Gas Dynamics: Theory, Techniques, and Applications. Birkhäuser, Boston, 2007.

[9] G. Karniadakis, A. Beskok, and N. Aluru. Microflows and Nanoflows. Springer, New York, 2004.

[10] P. L. Bhatnagar, E. P. Gross, and M. Krook. A model for collision processes in gases. I. Small amplitude processes in charged and neutral one-component systems. Phys. Rev., 94(3):511-525, 1954.

[11] P. Welander. On the temperature jump in a rarefied gas. Ark. Fys., 7:507-553, 1954.

[12] E. P. Gross and E. A. Jackson. Kinetic models and the linearized Boltzmann equation. Phys. Fluids, 2(4):432-441, 1959.

[13] D. R. Willis. Comparison of kinetic theory analyses of linearized Couette flow. Phys. Fluids, 5(2):127-135, 1962.

[14] S. Albertoni, C. Cercignani, and L. Gotusso. Numerical evaluation of the slip coefficient. Phys. Fluids, 6(7):993-996, 1963.

[15] C. Cercignani and C. D. Pagani. Variational approach to boundary-value problems in kinetic theory. Phys. Fluids, 9(6):1167-1173, 1966.

[16] C. Cercignani and F. Sernagiotto. Cylindrical Couette flow of a rarefied gas. Phys. Fluids, 10(6):1200-1204, 1967. 
[17] S. K. Loyalka. Velocity profile in Knudsen layer for Kramer's problem. Phys. Fluids, 18(12):1666-1669, 1975.

[18] S. K. Loyalka. Some exact numerical results for the BGK model: Couette, Poiseuille and thermal creep flow between parallel plates. Z. Angew. Math. Phys., 30(3):514-521, 1979.

[19] F. Coron. Derivation of slip boundary-conditions for the Navier-Stokes system from the Boltzmann equation. J. Stat. Phys., 54(3/4):829-857, Feb 1989.

[20] S. K. Loyalka and K. A. Hickey. The Kramer's problem: Velocity slip and defect for a hard sphere gas with arbitrary accommodation. $Z$. Angew. Math. Phys., 41(2):245-253, 1990.

[21] Y. Sone, S. Takata, and T. Ohwada. Numerical analysis of the plane Couette flow of a rarefied gas on the basis of the linearized Boltzmann equation for hard-sphere molecules. Euro. J. Mech. B/Fluids, 9(3):273288,1990 .

[22] F. Sharipov and V. Seleznev. Data on internal rarefied gas flows. J. Phys. Chem. Ref. Data, 27(3):657-706, May-Jun 1998.

[23] L. B. Barichello, M. Camargo, P. Rodrigues, and C. E. Siewert. Unified solutions to classical flow problems based on the BGK model. Z. Angew. Math. Phys., 52(3):517-534, May 2001.

[24] C. Cercignani, M. Lampis, and S. Lorenzani. Plane Poiseuille-Couette problem in micro-electro-mechanical systems applications with gasrarefaction effects. Phys. Fluids, 18(8):087102, Aug. 2006.

[25] S. K. Loyalka and R. V. Tompson. The velocity slip problem: Accurate solutions of the BGK model integral equation. Euro. J. Mech. B/Fluids, 28(2):211-213, 2009.

[26] F. Sharipov. Data on the velocity slip and temperature jump on a gassolid interface. J. Phys. Chem. Ref. Data, 40(2):023101, Jun 2011.

[27] L. Gibelli. Velocity slip coefficients based on the hard-sphere Boltzmann equation. Phys. Fluids, 24(2):022001, 2012. 
[28] Y. W. Yap and J. E. Sader. High accuracy numerical solutions of the Boltzmann Bhatnagar-Gross-Krook equation for steady and oscillatory Couette flows. Phys. Fluids, 24(3):032004, 2012.

[29] J. C. Maxwell. On stresses in rarified gases arising from inequalities of temperature. Philos. Trans. R. Soc. London, 170:231-256, 1879.

[30] L. Wu, C. White, T. J. Scanlon, J. M. Reese, and Y. Zhang. Deterministic numerical solutions of the Boltzmann equation using the fast spectral method. J. Comput. Phys., 250:27-52, Oct 12013.

[31] L. Wu, J. M. Reese, and Y. Zhang. Solving the Boltzmann equation deterministically by the fast spectral method: Application to gas microflows. J. Fluid Mech., 746:53-84, 2014.

[32] W. Li, L.-S. Luo, and J. Shen. Accurate solution and approximations of the linearized BGK equation for steady Couette flow. Comput. Fluids, 111:18-32, 2015.

[33] Y. W. Yap. Rarefied Gas Dynamics: Stokes' Second Problem. Honours thesis, The University of Melbourne, Victoria, Australia, 2009.

[34] A. J. MacLeod. Chebyshev expansion for Abramowitz functions. Appl. Numer. Math., 10:129-137, 1992.

[35] Y. Sone. Kinetic theory analysis of the linearized Rayleigh problem. Phys. Fluids, 7(3):470-471, 1964.

[36] M. Abramowitz and I. A. Stegun. Handbook of Mathematical Functions with Formulas, Graphs, and Mathematical Tables. National Bureau of Standards, Washington, DC, 1972.

[37] A. J. MacLeod. Chebyshev expansions for Abramowitz functions. Appl. Numer. Math., 10:129-137, 1992.

[38] I. S. Gradshteyn and I. M. Ryzbik. Table of Integrals, Series, and Products. Academic Press, New York, 6th edition, 2000.

[39] A. C. Pipkin. A Course on Integral Equations. Springer, New York, 1991. 
[40] G. B. Folland. Introduction to Partial Differential Equations. Princeton University Press, Princeton, 2nd edition, 1995.

[41] R. Kress. Linear Integral Equations, volume 82 of Applied Mathematics Sciences. Springer, New York, 3nd edition, 2014.

[42] D. R. Willis. Theoretical solutions to some nearly free molecular problems. In F. M. Devienne, editor, Rarefied Gas Dynamics: Proceedings of the First International Symposium held at Nice, International Series on Aeronautical Sciences and Space Flight, Division IX, Symposia, pages 246-257, New York, 1960. Pergammon.

[43] C. Cercignani. Plane Couette flow according to method of elementary solutions. J. Math. Anal. Appl., 11(1-3):93-101, 1965.

[44] D. R. Willis. A Study of Some Nearly Free Molecular Flow Problems. Phd thesis, Princeton University, Princeton, New Jersey, 1958.

[45] J. Ma, V. Rokhlin, and S. Wandzura. Generalized Gaussian quadrature rules for systems of arbitrary functions. SIAM J. Numer. Anal., 33(3):971-996, 1996.

[46] N. Yarvin and V. Rokhlin. Generalized Gaussian quadratures and singular value decompositions of integral operators. SIAM J. Sci. Comput., 20:699-718, 1998.

[47] J. Bremer, Z. Gimbutas, and V. Rokhlin. A nonlinear optimization procedure for generalized Gaussian quadratures. SIAM J. Sci. Comput., 32:1761-1788, 2010.

[48] J. Helsing. Solving integral equations on piecewise smooth boundaries using the RCIP method: A tutorial. Abstr. Appl. Anal., 2013:938167, 2013.

[49] J. Bremer. A fast direct solver for the integral equations of scattering theory on planar curves with corners. J. Comput. Phys., 231:1879-1899, 2012 .

[50] X. He and L.-S. Luo. A priori derivation of the lattice Boltzmann equation. Phys. Rev. E, 55(6):R6333-R6336, 1997. 
[51] X. He and L.-S. Luo. Theory of lattice Boltzmann method: From the Boltzmann equation to the lattice Boltzmann equation. Phys. Rev. E, 56(6):6811-6817, 1997.

[52] L.-S. Luo, M. Krafczyk, and W. Shyy. Lattice Boltzmann method for computational fluid dynamics. In R. Blockley and W. Shyy, editors, Encyclopedia of Arospace Engineering, chapter 56, pages 651-660. Wiley, New York, 2010.

[53] P. J. Dellar and L.-S. Luo. Lattice Boltzmann methods. In B. Engquist, editor, Encyclopedia of Applied and Computational Mathematics, pages 774-778. Springer, Berlin, 2015.

[54] M. Junk, A. Klar, and L.-S. Luo. Asymptotic analysis of the lattice Boltzmann equation. J. Comput. Phys., 210(2):676-704, 2005.

[55] L.-S. Luo. Comment on "Discrete Boltzmann equation for microfluidics". Phys. Rev. Lett., 92(13):139401, 2004.

[56] F. Verhaeghe, L.-S. Luo, and B. Blanpain. Lattice Boltzmann modeling of microchannel flow in slip flow regime. J. Comput. Phys., 228(1):147157, 2009 .

[57] T. Reis and P. J. Dellar. Lattice Boltzmann simulations of pressuredriven flows in micro channels using Navier-Maxwell slip boundary conditions. Phys. Fluids, 24(11):112001, 2012. 
Table A.5: The quadrature nodes and weights accurate to 20 digits for evaluating the integral of (A.1) with $\varphi\left(y ; x_{2}\right)$, and $x_{2}=0.674683166555077446339 \cdot 10^{-1}$. The total number of nodes is $N_{q}=35$.

\begin{tabular}{|c|c|}
\hline Nodes & Weights \\
\hline $0.792988988246045564030 \cdot 10^{-3}$ & $0.202688847471658432172 \cdot 10^{-2}$ \\
\hline $0.410693346378855415168 \cdot 10^{-2}$ & $0.455723617302932422087 \cdot 10^{-2}$ \\
\hline $0.978306191197271693046 \cdot 10^{-2}$ & $0.671625390675426981074 \cdot 10^{-2}$ \\
\hline $0.173528713722888959219 \cdot 10^{-1}$ & $0.831704746087239787293 \cdot 10^{-2}$ \\
\hline $0.261837431077278294910 \cdot 10^{-1}$ & $0.921966440226694785273 \cdot 10^{-2}$ \\
\hline $0.355326160136584480657 \cdot 10^{-1}$ & $0.934620975264074860619 \cdot 10^{-2}$ \\
\hline $0.446171252891122254754 \cdot 10^{-1}$ & $0.869883011423925961252 \cdot 10^{-2}$ \\
\hline $0.527033960605826855428 \cdot 10^{-1}$ & $0.737419903396351657245 \cdot 10^{-2}$ \\
\hline $0.592042475406610944382 \cdot 10^{-1}$ & $0.556881830803888309984 \cdot 10^{-2}$ \\
\hline $0.637755922727408659059 \cdot 10^{-1}$ & $0.356926002815703545700 \cdot 10^{-2}$ \\
\hline $0.663942224579436012947 \cdot 10^{-1}$ & $0.172474399663780336727 \cdot 10^{-2}$ \\
\hline $0.674040201036654659298 \cdot 10^{-1}$ & $0.460147654615133960566 \cdot 10^{-3}$ \\
\hline $0.681079848047553233655 \cdot 10^{-1}$ & $0.137995482404730805334 \cdot 10^{-2}$ \\
\hline $0.706774461128793896411 \cdot 10^{-1}$ & $0.398098325916874608230 \cdot 10^{-2}$ \\
\hline $0.765092149651476237298 \cdot 10^{-1}$ & $0.789603139400968518857 \cdot 10^{-2}$ \\
\hline $0.868744679127031704495 \cdot 10^{-1}$ & $0.130231305180939768305 \cdot 10^{-1}$ \\
\hline 0.102897602050623883257 & $0.191744061500602471763 \cdot 10^{-1}$ \\
\hline 0.125480544898040077161 & $0.260958838028227706484 \cdot 10^{-1}$ \\
\hline 0.155245827519492585278 & $0.334857239730032625968 \cdot 10^{-1}$ \\
\hline 0.192497505955192331980 & $0.410116417664372553736 \cdot 10^{-1}$ \\
\hline 0.237199035938706534720 & $0.483273909647583210614 \cdot 10^{-1}$ \\
\hline 0.288967164254755438563 & $0.550883119002058205810 \cdot 10^{-1}$ \\
\hline 0.347080881276407418032 & $0.609658877830082735527 \cdot 10^{-1}$ \\
\hline 0.410504369704090699004 & $0.656611444087150874148 \cdot 10^{-1}$ \\
\hline 0.477922670062561200450 & $0.689166353985062367955 \cdot 10^{-1}$ \\
\hline 0.547788496839131524923 & $0.705267052719613030729 \cdot 10^{-1}$ \\
\hline 0.618378325628786059291 & $0.703457181536915043453 \cdot 10^{-1}$ \\
\hline 0.687855573660713847516 & $0.682939740211733300377 \cdot 10^{-1}$ \\
\hline 0.754338447729138129358 & $0.643610980564879166826 \cdot 10^{-1}$ \\
\hline 0.815969860004009531002 & $0.586067733068871033977 \cdot 10^{-1}$ \\
\hline 0.870986730902716041798 & $0.511587859013520009042 \cdot 10^{-1}$ \\
\hline 0.917786022430558051086 & $0.422084651994775004976 \cdot 10^{-1}$ \\
\hline 0.954985001089820865331 & $0.320037662947611958015 \cdot 10^{-1}$ \\
\hline 0.981473704678799300941 & $0.208409070033924962209 \cdot 10^{-1}$ \\
\hline 0.996461532406522277493 & $0.906738134204675292845 \cdot 10^{-2}$ \\
\hline
\end{tabular}


Table A.6: The quadrature nodes and weights accurate to 20 digits for evaluating the integral of (A.1) with $\varphi\left(y ; x_{3}\right)$, and $x_{3}=0.160295215850487796882$. The total number of nodes is $N_{q}=38$.

\begin{tabular}{|c|c|}
\hline Nodes & Weights \\
\hline $0.132317303603991286984 \cdot 10^{-2}$ & $0.338607896886877699391 \cdot 10^{-2}$ \\
\hline $0.688781764995671268163 \cdot 10^{-2}$ & $0.769243850801178730792 \cdot 10^{-2}$ \\
\hline $0.165607996271652841987 \cdot 10^{-1}$ & $0.115594155044361594055 \cdot 10^{-1}$ \\
\hline $0.297813304783294696640 \cdot 10^{-1}$ & $0.147516725714038113474 \cdot 10^{-1}$ \\
\hline $0.457738457200097242314 \cdot 10^{-1}$ & $0.170746870426781268903 \cdot 10^{-1}$ \\
\hline $0.635920406213336210496 \cdot 10^{-1}$ & $0.183838794387567571368 \cdot 10^{-1}$ \\
\hline $0.821762970693130823770 \cdot 10^{-1}$ & $0.185998602957113710660 \cdot 10^{-1}$ \\
\hline 0.100426878513898443742 & $0.177243925603922427795 \cdot 10^{-1}$ \\
\hline 0.117292446268934881263 & $0.158544995669331342964 \cdot 10^{-1}$ \\
\hline 0.131869951647559002415 & $0.131901725366889318848 \cdot 10^{-1}$ \\
\hline 957934327981 & $0.100295095444753772230 \cdot 10^{-1}$ \\
\hline 0.151887646823574978616 & $0.674608424212412457708 \cdot 10^{-2}$ \\
\hline 0.157094076602884688241 & $0.374838172765476043519 \cdot 10^{-2}$ \\
\hline 69025306 & 0.14268297264412491835 \\
\hline 0.160380699641175804062 & $0.587108714793816953887 \cdot 10^{-3}$ \\
\hline 0.16178249945850777 & $0.249814696147291166432 \cdot 10^{-2}$ \\
\hline 3729525454445 & $0.581090078637114195641 \cdot 10^{-2}$ \\
\hline 0.1738079 & $0.103324788972480299403 \cdot 10^{-1}$ \\
\hline 0.186 & $0.158615243655112222074 \cdot 10^{-1}$ \\
\hline 0.205782408889073608 & $0.221434075282393075565 \cdot 10^{-1}$ \\
\hline 0.2312713 & $0.288853336173277607607 \cdot 10^{-1}$ \\
\hline 0.263 & $0.357708801533176429451 \cdot 10^{-1}$ \\
\hline 0.302752162337 & $0.424736095649928587625 \cdot 10^{-1}$ \\
\hline 0.348 & $0.486697990158569895380 \cdot 10^{-1}$ \\
\hline 0.39 & $0.540501071613229597711 \cdot 10^{-1}$ \\
\hline 0.456 & $0.583296778608032693826 \cdot 10^{-1}$ \\
\hline 0.516 & $0.612557479633713342327 \cdot 10^{-1}$ \\
\hline 0.5 & $0.626111766495475162742 \cdot 10^{-1}$ \\
\hline 0.640 & $0.622116809656615711119 \cdot 10^{-1}$ \\
\hline 0.701 & $0.598976346117463723572 \cdot 10^{-1}$ \\
\hline & $0.555545177284139263166 \cdot 10^{-1}$ \\
\hline 0.812367307968 & $0.493716991353965030022 \cdot 10^{-1}$ \\
\hline 0.85 & $0.427009011634395625885 \cdot 10^{-1}$ \\
\hline 0.8 & $0.374891022052934572338 \cdot 10^{-1}$ \\
\hline 34674402922217589 & $0.325824277533925632389 \cdot 10^{-1}$ \\
\hline 0.962831064670363947078 & $0.258545847308522730034 \cdot 10^{-1}$ \\
\hline 0.984522670061373795424 & $0.172806666173275645228 \cdot 10^{-1}$ \\
\hline 0.997026838077532786156 & $0.760898361372283415004 \cdot 10^{-2}$ \\
\hline
\end{tabular}


Table A.7: The quadrature nodes and weights accurate to 20 digits for evaluating the integral of (A.1) with $\varphi\left(y ; x_{4}\right)$, and $x_{4}=0.283302302935376404600$. The total number of nodes is $N_{q}=40$.

\begin{tabular}{|c|c|}
\hline Nodes & Weights \\
\hline $0.173460461230861797809 \cdot 10^{-2}$ & $0.444203888574733390124 \cdot 10^{-2}$ \\
\hline $0.905638645791270743456 \cdot 10^{-2}$ & $0.101521351822684414625 \cdot 10^{-1}$ \\
\hline $0.218929297351400672203 \cdot 10^{-1}$ & $0.154271292126592994411 \cdot 10^{-1}$ \\
\hline $0.396849015491781952071 \cdot 10^{-1}$ & $0.200258899432070901369 \cdot 10^{-1}$ \\
\hline $0.616500622720308964094 \cdot 10^{-1}$ & $0.237419543647182274221 \cdot 10^{-1}$ \\
\hline $0.868177636366624965546 \cdot 10^{-1}$ & $0.264064476050957009749 \cdot 10^{-1}$ \\
\hline 0.114071213278291167832 & $0.278976175248944942231 \cdot 10^{-1}$ \\
\hline 0.142199660212638115758 & $0.281510329769490535138 \cdot 10^{-1}$ \\
\hline 0.169960944844199036999 & $0.271700424201199224183 \cdot 10^{-1}$ \\
\hline 0.196154310254600065025 & $0.250355885035658718646 \cdot 10^{-1}$ \\
\hline 0.219701794532530809062 & $0.219129992559469165346 \cdot 10^{-1}$ \\
\hline 0.239733206455837725611 & $0.180515636444853887068 \cdot 10^{-1}$ \\
\hline 0.255665564874925629019 & $0.137741888768613335720 \cdot 10^{-1}$ \\
\hline 0.267275151441395511674 & $0.948463250419617489358 \cdot 10^{-2}$ \\
\hline 0.274844960981603345082 & $0.584282413250562047346 \cdot 10^{-2}$ \\
\hline 0.279437074501967613179 & $0.354832382584405186415 \cdot 10^{-2}$ \\
\hline 0.282127512400190904936 & $0.185018511750478201944 \cdot 10^{-2}$ \\
\hline 0.283230465637614619244 & $0.508676620974254980959 \cdot 10^{-3}$ \\
\hline 0.283985288812163377920 & $0.145794964662215488698 \cdot 10^{-2}$ \\
\hline 0.286652950737619037912 & $0.408199014781586548936 \cdot 10^{-2}$ \\
\hline 0.292537057737346286183 & $0.786660958841799449886 \cdot 10^{-2}$ \\
\hline 0.302713486761914568685 & $0.126314849813659375353 \cdot 10^{-1}$ \\
\hline 0.318049414104770388345 & $0.181433591919002659863 \cdot 10^{-1}$ \\
\hline 0.339159994288200992045 & $0.241338959666198596476 \cdot 10^{-1}$ \\
\hline 0.366380320470736459203 & $0.303134919122624470235 \cdot 10^{-1}$ \\
\hline 50559379603292058 & $0.363839476609952488678 \cdot 10^{-1}$ \\
\hline 0.439013407884023057366 & $0.420504767904759100672 \cdot 10^{-1}$ \\
\hline 0.483623266211056455235 & $0.470331195253690897493 \cdot 10^{-1}$ \\
\hline 766471367203932271 & $0.510774128267439327650 \cdot 10^{-1}$ \\
\hline 0.585391722701490026486 & $0.539640556216762704817 \cdot 10^{-1}$ \\
\hline 0.640249543446034032165 & $0.555172637456027239608 \cdot 10^{-1}$ \\
\hline 0.69593931540222693 & $0.556115134367986796837 \cdot 10^{-1}$ \\
\hline 0.750962139630901539335 & $0.541764106396923673505 \cdot 10^{-1}$ \\
\hline 0.803777542649091509574 & $0.511994853404870936539 \cdot 10^{-1}$ \\
\hline 0.852861884617007327308 & $0.467267877351197161327 \cdot 10^{-1}$ \\
\hline 0.896766245954353142497 & $0.408612507483427623257 \cdot 10^{-1}$ \\
\hline 0.934171581034525453279 & $0.337588811446719678002 \cdot 10^{-1}$ \\
\hline 0.963939051660569106661 & $0.256229796872352476133 \cdot 10^{-1}$ \\
\hline 0.985153838623386467045 & $0.166971285400753536986 \cdot 10^{-1}$ \\
\hline 0.997163901020594249056 & $0.726723452416515237665 \cdot 10^{-2}$ \\
\hline
\end{tabular}


Table A.8: The quadrature nodes and weights accurate to 20 digits for evaluating the integral of (A.1) with $\varphi\left(y ; x_{5}\right)$, and $x_{5}=0.425562830509184394557$. The total number of nodes is $N_{q}=40$.

\begin{tabular}{|c|c|}
\hline Nodes & Weights \\
\hline $0.220881298951836319454 \cdot 10^{-2}$ & $0.565798259230912136519 \cdot 10^{-2}$ \\
\hline $0.115459857740097991261 \cdot 10^{-1}$ & $0.129623232105893566642 \cdot 10^{-1}$ \\
\hline $0.279721655410615419585 \cdot 10^{-1}$ & $0.197861700713755003553 \cdot 10^{-1}$ \\
\hline $0.508683607973689484739 \cdot 10^{-1}$ & $0.258612260323197713517 \cdot 10^{-1}$ \\
\hline $0.793681812770212477592 \cdot 10^{-1}$ & $0.309582931339356466059 \cdot 10^{-1}$ \\
\hline 0.112395266226514324587 & $0.348879072092039449756 \cdot 10^{-1}$ \\
\hline 0.148706941594180936498 & $0.375081919986768758780 \cdot 10^{-1}$ \\
\hline 0.186945529539370016057 & $0.387321641615741159062 \cdot 10^{-1}$ \\
\hline 0.225696559718626868981 & $0.385342512285009032021 \cdot 10^{-1}$ \\
\hline 0.263553171083603428627 & $0.369561770912352679286 \cdot 10^{-1}$ \\
\hline 0.299186081506572750608 & $0.341121589336557728929 \cdot 10^{-1}$ \\
\hline 0.331418120380714103658 & $0.301926046211423893513 \cdot 10^{-1}$ \\
\hline 0.359300876689618204840 & $0.254641538720032349616 \cdot 10^{-1}$ \\
\hline 0.382188095912621534211 & $0.202624471047822631237 \cdot 10^{-1}$ \\
\hline 0.399796540766484022772 & $0.149737446015835978199 \cdot 10^{-1}$ \\
\hline 0.412242123734758766882 & $0.100039894339543994231 \cdot 10^{-1}$ \\
\hline 0.420040124766145508381 & $0.573900047785795780770 \cdot 10^{-2}$ \\
\hline 0.424064641212417326428 & $0.250448505771265722099 \cdot 10^{-2}$ \\
\hline 0.425476202122843967303 & $0.595188164999202215841 \cdot 10^{-3}$ \\
\hline 57639994588051498 & $0.144415166138138017577 \cdot 10^{-2}$ \\
\hline 467892617383913 & $0.374187291225132148322 \cdot 10^{-2}$ \\
\hline 0.433956725600945 & $0.669951567565831870245 \cdot 10^{-2}$ \\
\hline 311276144982553 & $0.103764116733157722975 \cdot 10^{-1}$ \\
\hline 0.455 & $0.150470818083990153032 \cdot 10^{-1}$ \\
\hline 047145840508029 & $0.205281582784439395470 \cdot 10^{-1}$ \\
\hline 28703786875145 & $0.263205549766694685260 \cdot 10^{-1}$ \\
\hline 0.52 & $0.319664321345156259763 \cdot 10^{-1}$ \\
\hline 725318 & $0.370995271522314637691 \cdot 10^{-1}$ \\
\hline 142711 & $0.414209323301688292590 \cdot 10^{-1}$ \\
\hline 30204 & $0.446890735084800862935 \cdot 10^{-1}$ \\
\hline 2227 & $0.467184380181242376876 \cdot 10^{-1}$ \\
\hline 0.735438898260041017363 & $0.473802819966573597095 \cdot 10^{-1}$ \\
\hline 046209081217463 & $0.466033124222897930302 \cdot 10^{-1}$ \\
\hline 0.828160689205015481920 & $0.443737299430293501701 \cdot 10^{-1}$ \\
\hline 0.870828931146946361745 & $0.407342333599802829317 \cdot 10^{-1}$ \\
\hline 0.909190901123286251383 & $0.357816514215000792679 \cdot 10^{-1}$ \\
\hline 0.942003042264273567793 & $0.296629865992029448124 \cdot 10^{-1}$ \\
\hline 0.968191628716285478882 & $0.225698458556575725965 \cdot 10^{-1}$ \\
\hline 0.986894065476221026923 & $0.147317819186105453745 \cdot 10^{-1}$ \\
\hline 0.997495231223995566697 & $0.641756735602063403527 \cdot 10^{-2}$ \\
\hline
\end{tabular}

